\title{
Dynamical Performances of a Vibration Absorber for Continuous Structure considering Time-Delay Coupling
}

\author{
Xiuting Sun and Youshuo Song \\ School of Mechanical Engineering, University of Shanghai for Science and Technology, Shanghai 200093, China \\ Correspondence should be addressed to Youshuo Song; wssys2006@163.com
}

Received 20 November 2015; Accepted 15 June 2016

Academic Editor: Ivo Caliò

Copyright (c) 2016 X. Sun and Y. Song. This is an open access article distributed under the Creative Commons Attribution License, which permits unrestricted use, distribution, and reproduction in any medium, provided the original work is properly cited.

\begin{abstract}
The nonlinear effect incurred by time delay in vibration control is investigated in this study via a vibration absorber coupled with a continuous beam structure. The stability of the vibration absorber coupled structure system with time-delay coupling is firstly studied, which provides a general guideline for the potential time delay to be introduced to the system. Then it is shown that there is a specific region for the time delay which can bring bifurcation modes to the dynamic response of the coupling system, and the vibration energy at low frequencies can be transferred or absorbed due to the bifurcation mode and the vibration in the corresponding frequency range is thus suppressed. The nonlinear mechanism of this vibration suppression incurred by the coupling time delay is discussed in detail, which provides a novel and alternative approach to the analysis, design, and control of vibration absorbers in engineering practice.
\end{abstract}

\section{Introduction}

Vibration absorbers including passive and active dynamic vibration absorbers received increasing attention in structural vibration control in the past several years [1-5]. The mechanism of a passive vibration absorber is to distribute the vibration energy through coupling a slave structure to the master one [6], while an active vibration absorber produces forces to counteract the vibration through adjusting the slave structure according to the motion state of the master [7].

Considering the mechanism of various vibration absorbers developed in the literature, many different works have been done, which provide a fundamental basis for the understanding of nonlinear dynamics in vibration control. Wong and Cheung [8] employed the damping characteristics of a vibration absorber to control the master system by dissipating the vibration energy with the damper of the absorber. Kim et al. [9] considered optimization of the damping characteristics of vibration absorbers. The optimal damping ratio for the master and slave structure under study is therefore obtained so that the amplitude of the master structure can be kept in a small range. Natsiavas [10] investigated vibration absorbers with nonlinear springs since soft and hard springs have very different effect on system damping characteristics. However, it is noticed that passive vibration absorbers are often unsatisfactory for their uncontrolled reaction under complex working conditions in practice. Huang et al. [11] used a servo motor as actuator to drive a vibration absorber and achieve remarkable vibration suppression performance. Kobori et al. [12] proposed a concept of active variable stiffness in active vibration control and explored the potential benefits of nonlinear dynamics incurred by time delay in vibration control.

Noticeably, $\mathrm{Xu}$ et al. $[13,14]$ presented that time delay could be used as a simple but efficient "switch" to control motions of a system for different applications, which was proved by relative experiment of a linear time-delayed control absorber. A delayed-resonator vibration absorber is first proposed by Olgac et al. [15-17]. Vibration energy of a linear master structure is absorbed to suppress undesired oscillations by coupling a time delay to a master mass. To broaden a valid range in frequency for suppression of vibrations, Zhao and $\mathrm{Xu}$ [18] proposed a delayed-resonator vibration absorber with nonlinear master and slave structures, and then a linear timedelayed active absorber was realized in relative experiment $[19,20]$. The slave structure in this study has geometrical nonlinearity, which can expand the frequency range so that vibration energy could be absorbed easily in engineering 
practice. It is shown that the amplitude of the master system is decreased by at least $10 \%$ as compared to that without the time-delayed feedback control, and the complex dynamics of the system induced by the time delay are also preliminarily studied. Sun et al. [14,21] utilized linear and nonlinear velocity feedback to control a continuous beam for parametrical resonance. It is shown that the active control designed suppresses the parametric resonance for force excitation in the theoretical analysis. The significant effect of the vibration control has been proved and the bifurcation in parametric excitation system can be eliminated in the related experiment. In previous studies, the complex nonlinear dynamics induced by time delay are usually considered to be harmful for structural vibration control. However, it is more and more noticed that some predicable nonlinear dynamics could have beneficial influence in vibration control, due to the fact that multiple vibration modes could result in energy transmission or suppression under certain conditions. Although some preliminary results have shown that the nonlinear dynamics incurred by time delay in the coupling of slave and master structures could be advantageous in absorbing vibration energy, a systematic and theoretical study on the nonlinear mechanism in affecting the stability of continuous structures and absorbing vibration energy is still yet to be further developed.

In this paper, to investigate the nonlinear mechanism of vibration absorbers with time delays, a vibration absorber system (VAS) under harmonic excitation is studied. The VAS includes a continuous beam structure as the master structure and a continuous structure consisting of two beams as the slave system, which also acts as an absorber. After coupling the master structure and the slave system with a spring and a servo motor, there exists energy transmission between these two structures [22]. Basically, the geometry of the slave structure and the coupling strength should be carefully designed so that the frequency of vibration modes bifurcated by the introduced time delay could be proportional to the excitation frequency. In this way, it is known that the coupled modes of nonlinear systems can realize energy transmission and possible suppression [23]. The slave structure in this study has geometrical nonlinearity, which can expand the frequency range where vibration energy could be absorbed [24]. In the present research, it is shown that there is a specific region for the time delay which can bring bifurcation modes to the dynamic response of the system, while the latter can absorb the vibration energy at low frequencies and thus suppress the vibration over the corresponding frequency range. The nonlinear mechanism of this vibration suppression incurred by the coupling time delay is discussed in detail. This provides a novel and alternative insight into the analysis, design, and control of vibration absorbers in engineering practice.

The paper is organized as follows. Firstly, the continuous structure system is modeled and the nonlinear dynamics of both master and slave structures are considered in Section 2. The relationship between the coupling spring stiffness and the amplitude of the master structure is derived. Secondly, the nonlinear effect induced by the time delay via the servo motor is studied. The stability region is determined through the eigenvalues of vibration equations in Section 3. Owing to the stability of fixed point, the master structure is under periodic motion which can be described by Harmonic Balance Method (HBM) $[25,26]$. Complex dynamical behavior would appear because of the bifurcation induced by time delay. The beneficial region of the time delay for vibration suppression is developed in Section 4. The quasi-periodic solution is solved by Perturbation Incrementation Scheme (PIS) $[27,28]$ and Multiscale Method (MS) [29]. The nonlinear mechanism of the vibration absorber under different regions of the time delay is also discussed in Section 4. A conclusion is given in Section 5.

\section{The Nonlinear Vibration and Its Modeling}

The system under consideration is shown schematically in Figure 1. The master (Beam 1) and the slave structures (Beams 2 and 3) are coupled by two springs and a servo motor since the slave structure may perform a geometrically nonlinear nature [24], where $k_{3}$ and $k_{\alpha}$ are the linear stiffness of coupling and controllable springs, respectively. The servo motor is used to provide the strength of $k_{\alpha}$ and the coupling delay in demand so that $k_{\alpha}$ and the delay are considered as two controllable parameters to suppress or absorb vibrations of the master structure. It should be noted that the equivalent mass of the slave structure is much smaller than the master structure for the convenience of the installation and realization. The geometric sizes of the three beams are indicated in Figure 1 and the lengths of Beams 1,2 , and 3 are $l_{1}=1 \mathrm{~m}$, $l_{2}=0.4 \mathrm{~m}$, and $l_{3}=0.42 \mathrm{~m}$, respectively. The material of the model is steel, and Young's modulus of the material is $E=$ $200 \mathrm{GPa}$.

The state based actuator in the control spring by the servo motor is given by $w_{1}\left(l_{1} / 2, t-\tau_{k}\right)-w_{2}\left(l_{2} / 2, t-\tau_{k}\right)$ with time delay $\tau_{k}$. The potential damping effect of the time delay would be focused for this control strategy. The dynamical equations of the continuous structure with the actuator are

$$
\begin{aligned}
& \rho_{l_{1}} \frac{\partial^{2} w_{1}}{\partial t^{2}}+E I_{1} \frac{\partial^{4} w_{1}}{\partial s_{1}^{4}}+C_{1} \frac{\partial w_{1}}{\partial t}-\frac{E A_{1}}{2 l_{1}} \\
& \quad \int_{0}^{l_{1}}\left(\frac{\partial w_{1}}{\partial s_{1}}\right)^{2} d s_{1} \frac{\partial^{2} w_{1}}{\partial s_{1}^{2}} \\
& \quad=k_{\alpha}\left[w_{1}\left(\frac{l_{1}}{2}, t-\tau_{k}\right)-w_{2}\left(\frac{l_{2}}{2}, t-\tau_{k}\right)\right. \\
& \left.\quad+w_{2}\left(\frac{l_{2}}{2}, t\right)-w_{1}\left(\frac{l_{1}}{2}, t\right)\right] \delta\left(s_{1}-\frac{l_{1}}{2}\right) \\
& \quad+\left\{k_{3}\left[w_{2}\left(\frac{l_{2}}{2}, t\right)-w_{1}\left(\frac{l_{1}}{2}, t\right)\right]+F \cos \omega t\right\} \delta\left(s_{1}\right. \\
& \left.\quad-\frac{l_{1}}{2}\right), \\
& \left(\rho_{l_{2}} \frac{\partial^{2} w_{2}}{\partial t^{2}}+E I_{2} \frac{\partial^{4} w_{2}}{\partial s_{2}^{4}}+C_{2} \frac{\partial w_{2}}{\partial t}\right. \\
& \left.\quad-\frac{E A_{2}}{2 l_{2}} \int_{0}^{l_{2}}\left(\frac{\partial w_{2}}{\partial s_{2}}\right)^{2} d s_{2} \frac{\partial^{2} w_{2}}{\partial s_{2}^{2}}\right) \delta\left(s_{3}-l_{3}\right)
\end{aligned}
$$




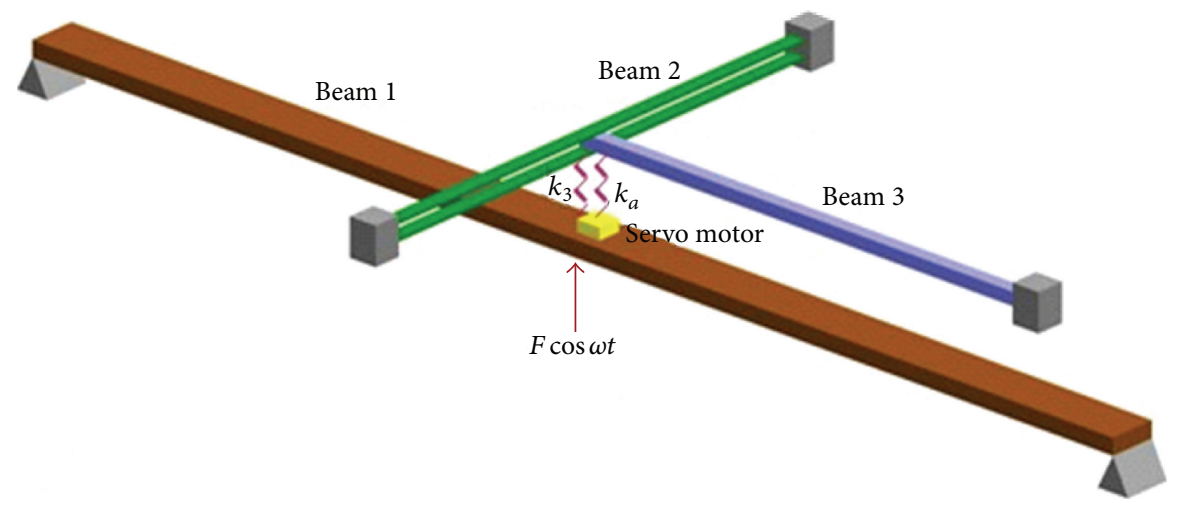

(a)
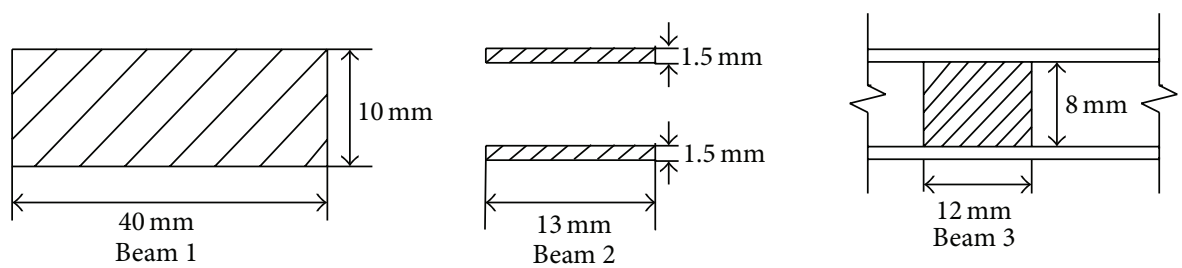

(b)

FIGURE 1: The schematic diagram of the structure: (a) the scheme of structure; (b) the section size of each beam.

$$
\begin{aligned}
& +\left(\rho_{l_{3}} \frac{\partial^{2} w_{3}}{\partial t^{2}}+E I_{3} \frac{\partial^{4} w_{3}}{\partial s_{3}^{4}}+C_{3} \frac{\partial w_{3}}{\partial t}\right. \\
& \left.-\frac{E A_{3}}{2 l_{3}} \int_{0}^{l_{3}}\left(\frac{\partial w_{3}}{\partial s_{3}}\right)^{2} d s_{3} \frac{\partial^{2} w_{3}}{\partial s_{3}^{2}}\right) \delta\left(s_{2}-\frac{l_{2}}{2}\right) \\
& =k_{\alpha}\left[w_{2}\left(\frac{l_{2}}{2}, t-\tau_{k}\right)-w_{1}\left(\frac{l_{1}}{2}, t-\tau_{k}\right)\right. \\
& \left.+w_{1}\left(\frac{l_{1}}{2}, t\right)-w_{2}\left(\frac{l_{2}}{2}, t\right)\right] \delta\left(s_{3}-l_{3}\right) \delta\left(s_{2}-\frac{l_{2}}{2}\right) \\
& +k_{3}\left[w_{1}\left(\frac{l_{1}}{2}, t\right)-w_{2}\left(\frac{l_{2}}{2}, t\right)\right] \delta\left(s_{3}-l_{3}\right) \delta\left(s_{2}\right. \\
& \left.-\frac{l_{2}}{2}\right) .
\end{aligned}
$$

All the parameters in the equations above such as $k_{\alpha}, k_{3}$, $E, A_{1}, A_{2}$, and $A_{3}$ are defined in Physical Parameters of the Structure. The deflections of each beam $w_{i}\left(s_{i}, t\right)$ are $w_{1}=\sum_{i=1}^{n} z_{1 i}(t) \phi_{1 i}\left(s_{1}\right), w_{2}=\sum_{i=1}^{n} z_{2 i}(t) \phi_{2 i}\left(s_{2}\right)$, and $w_{3}=$ $\sum_{i=1}^{n} z_{3 i}(t) \phi_{3 i}\left(s_{3}\right)$. The high order modes are ignored due to our goal to suppress or absorb the buckling vibration in the present research. Therefore, the dynamics of the structure are truncated to the first mode as $w_{1}=z_{11}(t) \phi_{11}\left(s_{1}\right), w_{2}=$ $z_{21}(t) \phi_{21}\left(s_{2}\right)$, and $w_{3}=z_{31}(t) \phi_{31}\left(s_{3}\right)$. The first mode of each beam in the structure is determined by boundary conditions. The master beam (Beam 1 ) is a simply supported beam at both ends and Beam 2 is fixed at both ends. Beam 3 is fixed at one end and is fixed to the midpoint of Beam 2 at another end, which provides a boundary condition by deformation compatibility with the midpoint of Beam 2. The first mode of each beam is given as follows:

$$
\begin{aligned}
& \phi_{11}\left(s_{1}\right)=a_{1} \sin \frac{\pi s_{1}}{l_{1}}, \\
& \phi_{21}\left(s_{2}\right)=a_{2}\left(\cos \beta_{21} s_{2}-\cosh \beta_{21} s_{2}\right. \\
& \left.\quad-\frac{\cos \beta_{21} l_{2}-\cosh \beta_{21} l_{2}}{\sin \beta_{21} l_{2}-\sinh \beta_{21} l_{2}}\left(\sin \beta_{21} s_{2}-\sinh \beta_{21} s_{2}\right)\right), \\
& \phi_{31}\left(s_{3}\right)=a_{3}\left(\cos \beta_{31} s_{3}-\cosh \beta_{31} s_{3}\right. \\
& \left.-\frac{\cos \beta_{31} l_{3}+\cosh \beta_{31} l_{3}}{\sin \beta_{31} l_{3}+\sinh \beta_{31} l_{3}}\left(\sin \beta_{31} s_{3}-\sinh \beta_{31} s_{3}\right)\right) .
\end{aligned}
$$

Utilizing the compatible displacement $w_{2}\left(l_{2} / 2, t\right)=$ $w_{3}\left(l_{3}, t\right)$, the coefficients of mode equations above can be computed as $a_{1}=1, a_{2}=1 / 1.58815$, and $a_{3}=1 / 1.99985$, and the system is a two-degree-of-freedom vibration system because of the compatibility condition of Beam 2 and Beam 3. The vibration equations can then be obtained as

$$
\begin{aligned}
m_{1} \ddot{z}_{1} & +k_{1} z_{1}+c_{1} \dot{z}_{1}+k k_{1} z_{1}^{3} \\
= & k_{3}\left(z_{2}-z_{1}\right)+k_{\alpha}\left[\left(z_{1 \tau}-z_{2 \tau}\right)-\left(z_{1}-z_{2}\right)\right] \\
& \quad+F \cos (\omega t) \\
& m_{2} \ddot{z}_{2}+k_{2} z_{2}+c_{2} \dot{z}_{2}+k k_{2} z_{2}^{3} \\
= & k_{3}\left(z_{1}-z_{2}\right)-k_{\alpha}\left[\left(z_{1 \tau}-z_{2 \tau}\right)-\left(z_{1}-z_{2}\right)\right],
\end{aligned}
$$


where

$$
\begin{aligned}
m_{1}= & \rho_{l_{1}} \int_{0}^{l_{1}} \phi_{11}^{2}\left(s_{1}\right) d s_{1}, \\
k_{1}= & E I_{1} \int_{0}^{l_{1}} \phi_{11}^{(4)}\left(s_{1}\right) \phi_{11}\left(s_{1}\right) d s_{1}, \\
c_{1}= & C_{1} \int_{0}^{l_{1}} \phi_{11}^{2}\left(s_{1}\right) d s_{1}, \\
m_{2}= & \rho_{l_{2}} \int_{0}^{l_{2}} \phi_{21}^{2}\left(s_{2}\right) d s_{2}+\rho_{l_{3}} \int_{0}^{l_{3}} \phi_{31}^{2}\left(s_{3}\right) d s_{3}, \\
c_{2}= & C_{2} \int_{0}^{l_{2}} \phi_{21}^{2}\left(s_{2}\right) d s_{2}+C_{3} \int_{0}^{l_{3}} \phi_{31}^{2}\left(s_{3}\right) d s_{3}, \\
k k_{1}= & \frac{E A_{1}}{2 l_{1}} \int_{0}^{l_{1}} \int_{0}^{l_{1}} \phi_{11}^{\prime 2}\left(s_{1}\right) d s_{1} \phi_{11}^{\prime \prime}\left(s_{1}\right) \phi_{11}\left(s_{1}\right) d s_{1}, \\
k_{2}= & E I_{2} \int_{0}^{l_{2}} \phi_{21}^{(4)}\left(s_{2}\right) \phi_{21}\left(s_{2}\right) d s_{2} \\
& +E I_{3} \int_{0}^{l_{3}} \phi_{31}^{(4)}\left(s_{3}\right) \phi_{31}\left(s_{3}\right) d s_{3}, \\
f= & F \phi_{11}\left(\frac{l_{1}}{2}\right), \\
& +\frac{E A_{2}}{2 l_{3}} \int_{0}^{l_{2}} \int_{0}^{l_{2}} \phi_{21}^{\prime 2}\left(s_{2}\right) d s_{2} \phi_{21}^{\prime \prime}\left(s_{2}\right) \phi_{21}\left(s_{2}\right) d s_{2} \\
k k_{2} & l_{31}^{\prime 2}\left(s_{3}\right) d s_{3} \phi_{31}^{\prime \prime}\left(s_{3}\right) \phi_{31}\left(s_{3}\right) d s_{3} .
\end{aligned}
$$

The values of the parameters calculated by (4) are listed in Table 1.

\section{Natural Frequency and Stability of the System}

3.1. Without Time Delay. The dynamic response of the system without time delay is considered firstly. The natural frequency of the structure can be obtained through solving the eigenvalue problem of the linear part of (3). The natural frequencies of the structure are defined as $\Omega_{1}$ and $\Omega_{2}$. Ignoring the damping effect, the frequencies can be solved by

$$
\left|\mathbf{K}-\Omega^{2} \mathbf{M}\right|=0,
$$

where the stiffness matrix

$$
\mathbf{K}=\left(\begin{array}{cc}
k_{1}+k_{3} & -k_{3} \\
-k_{3} & k_{2}+k_{3}
\end{array}\right)
$$

and the system mass matrix

$$
\mathbf{M}=\left(\begin{array}{cc}
m_{1} & 0 \\
0 & m_{2}
\end{array}\right) .
$$

The natural frequencies can be obtained as

$$
\begin{aligned}
& \Omega_{1}=\sqrt{\frac{k_{1}+k_{3}}{2 m_{1}}+\frac{k_{2}+k_{3}}{2 m_{2}}-\frac{1}{2} \sqrt{\left[\frac{k_{1}+k_{3}}{m_{1}}+\frac{k_{2}+k_{3}}{m_{2}}\right]^{2}+\frac{k_{1} k_{2}+k_{1} k_{3}+k_{2} k_{3}}{m_{1} m_{2}}}}, \\
& \Omega_{2}=\sqrt{\frac{k_{1}+k_{3}}{2 m_{1}}+\frac{k_{2}+k_{3}}{2 m_{2}}+\frac{1}{2} \sqrt{\left[\frac{k_{1}+k_{3}}{m_{1}}+\frac{k_{2}+k_{3}}{m_{2}}\right]^{2}+\frac{k_{1} k_{2}+k_{1} k_{3}+k_{2} k_{3}}{m_{1} m_{2}}}} .
\end{aligned}
$$

The relationship between the natural frequencies $\Omega_{1}$ and $\Omega_{2}$ of the structure and the stiffness of coupling spring $k_{3}$ is shown in Figure 2. The coupling spring actually increases the natural frequency of the structure. This results in the first natural frequency of the master structure being moved to a higher frequency. The vibration amplitude of the master structure under different stiffness of the coupling spring $k_{3}$ is given in Figure 3. The vibration amplitude of the master structure decreases after the slave structure is coupled through the coupling spring.

Figure 3 shows that the coupling spring helps to suppress the vibration of the master structure. However, as the stiffness of the coupling spring increases from zero to $4000 \mathrm{~N} \cdot \mathrm{m}^{-1}$, the amplitude of the master structure decreases from $1.6 \mathrm{~mm}$ to
$1.1 \mathrm{~mm}$. The vibration suppression due to the coupling spring actually is not significant to meet engineering requirements. For vibration suppression effect, a time-delayed active control is proposed in the following section via the servo motor, which makes the slave structure a much more powerful vibration absorber. The time delay can be adjusted and controlled to give the best vibration suppression.

3.2. With Time Delay. Because of the unsatisfactory vibration suppression effect of the coupling spring, the time delay is proposed to be introduced in this section as a key element for performance improvement. Owing to the fact that the amplitude of the external excitation could be small, the small external excitation in this paper cannot cause complex 
TABLE 1: The value of physical parameters.

\begin{tabular}{lc}
\hline Physical parameters & Value \\
\hline$m_{1}$ & 1.57 \\
$k_{1}$ & 34093.2 \\
$m_{2}$ & 0.127676 \\
$k_{2}$ & 9247.18 \\
$k k_{1}$ & $1.0228 \times 10^{9}$ \\
$k k_{2}$ & $1.48863 \times 10^{9}$ \\
$c_{1}$ & 12 \\
$c_{2}$ & 0.75 \\
\hline
\end{tabular}

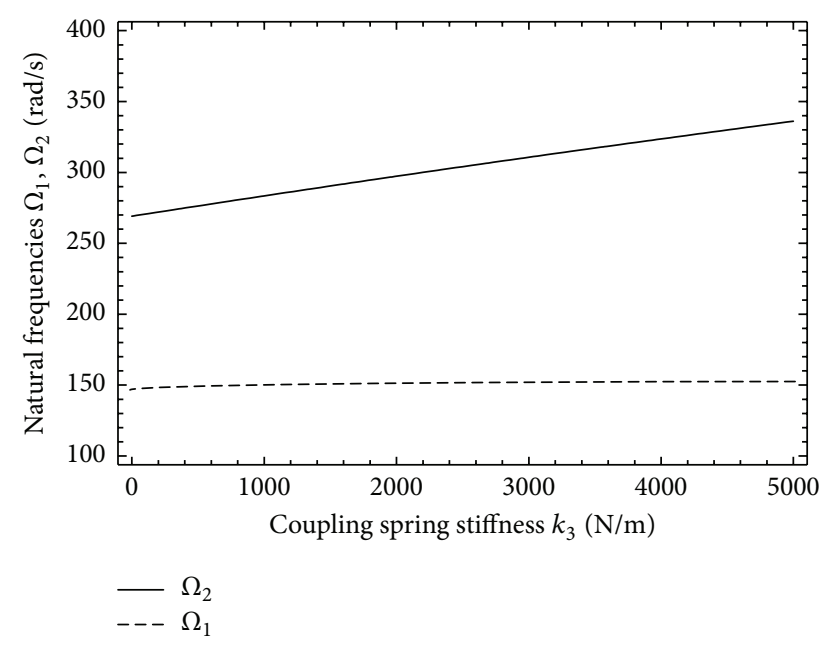

FIgURE 2: The natural frequencies of the structure without time delay.

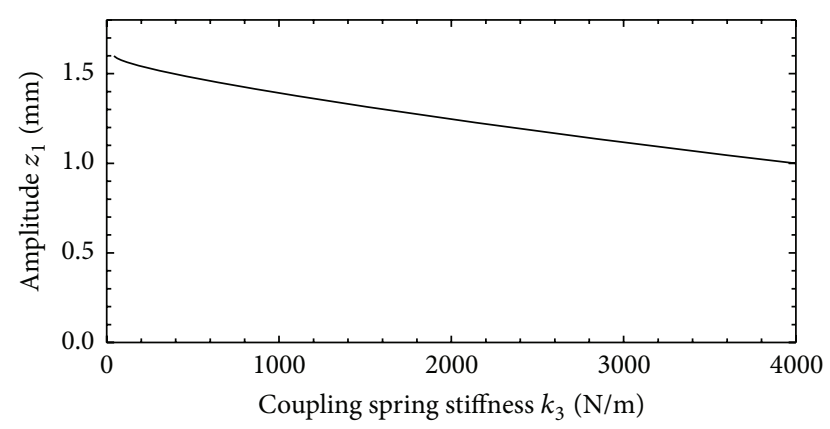

FIGURE 3: The amplitude of master beam and the stiffness of coupling spring as $F=3 \mathrm{~N}$ and $\omega=149 \mathrm{rad} / \mathrm{s}$.

dynamics in that the magnitude of the external excitation is much smaller than other terms in the equation, but the nonlinearity and the time delay could induce bifurcation and even unstable dynamics, which should be systematically investigated.
Introducing the following dimensionless variables,

$$
\begin{aligned}
& \Omega^{\prime}=\sqrt{\frac{k_{1}}{m_{1}}}, \\
& t^{\prime}=\Omega^{\prime} t, \\
& \tau=\Omega^{\prime} \tau_{k}, \\
& \omega^{\prime}=\frac{\omega}{\Omega^{\prime}}, \\
& \omega_{1}^{\prime}=1, \\
& \xi_{1}=\frac{c_{1}}{\Omega^{\prime} m_{1}}, \\
& \gamma_{1}=\frac{k k_{1}}{k_{1}}, \\
& \alpha_{1}=\frac{k_{3}}{k_{1}}, \\
& g_{1}=\frac{k_{\alpha}}{k_{1}} \text {, } \\
& \omega_{2}^{\prime}=\sqrt{\frac{m_{1} k_{2}}{m_{2} k_{1}}}, \\
& \xi_{2}=\frac{c_{2}}{\Omega^{\prime} m_{2}} \text {, } \\
& \gamma_{2}=\frac{m_{1} k k_{2}}{m_{2} k_{1}}, \\
& \alpha_{2}=\frac{m_{1} k_{3}}{m_{2} k_{1}}, \\
& g_{2}=\frac{m_{1} k_{\alpha}}{m_{2} k_{1}}, \\
& f=\frac{F}{k_{1}},
\end{aligned}
$$

then the vibration equations can be rewritten as

$$
\begin{aligned}
\ddot{z}_{1}+ & \omega_{1}^{\prime 2} z_{1}+\xi_{1} \dot{z}_{1}+\gamma_{1} z_{1}^{3} \\
= & \alpha_{1}\left(z_{2}-z_{1}\right)+g_{1}\left[\left(z_{1 \tau}-z_{2 \tau}\right)-\left(z_{1}-z_{2}\right)\right] \\
& +f \cos \left(\omega^{\prime} t^{\prime}\right) \\
& \quad \\
\ddot{z}_{2}+ & \omega_{2}^{\prime 2} z_{2}+\xi_{2} \dot{z}_{2}+\gamma_{2} z_{2}^{3} \\
= & \alpha_{2}\left(z_{1}-z_{2}\right)-g_{2}\left[\left(z_{1 \tau}-z_{2 \tau}\right)-\left(z_{1}-z_{2}\right)\right] .
\end{aligned}
$$

For the values of dimensionless parameters, please refer to Table 2 . 
TABLE 2: The value of dimensionless parameters.

\begin{tabular}{lc}
\hline Dimensionless parameters & Value \\
\hline$\Omega^{\prime}$ & 147.361 \\
$\omega_{1}^{\prime}$ & 1 \\
$\omega_{2}^{\prime}$ & 1.82629 \\
$\gamma_{1}$ & 30000 \\
$\gamma_{2}$ & 549157.6 \\
$\xi_{1}$ & 0.045 \\
$\xi_{1}$ & 0.03 \\
$\Omega_{1}^{\prime}$ & $\Omega_{1} / 147.36$ \\
$\tau$ & $147.36 \tau_{k}$ \\
$\alpha_{1}$ & $2.933 \times 10^{-5} k_{3}$ \\
$\alpha_{2}$ & $3.6068 \times 10^{-4} k_{3}$ \\
$g_{1}$ & $2.933 \times 10^{-5} k_{\alpha}$ \\
$g_{2}$ & $3.6068 \times 10^{-4} k_{\alpha}$ \\
$f$ & $2.933 \times 10^{-5} F$ \\
$\omega^{\prime}$ & $\omega / 147.36$ \\
$\Omega_{2}^{\prime}$ & $\Omega_{2} / 147.36$ \\
\hline
\end{tabular}

After setting $\left\{z_{1}, y_{1}, z_{2}, y_{2}\right\}^{T}=\left\{z_{1}, \dot{z}_{1}, z_{2}, \dot{z}_{2}\right\}^{T}$, the linear part of the dimensionless vibration equation can be written as

$$
\begin{aligned}
\dot{z}_{1}= & y_{1}, \\
\dot{y}_{1}= & -\omega_{1}^{\prime 2} z_{1}-\xi_{1} y_{1}+\alpha_{1}\left(z_{2}-z_{1}\right) \\
& +g_{1}\left[\left(z_{1 \tau}-z_{2 \tau}\right)-\left(z_{1}-z_{2}\right)\right], \\
\dot{z}_{2}= & y_{2}, \\
\dot{y}_{2}= & -\omega_{2}^{\prime 2} z_{2}-\xi_{2} y_{2}-\alpha_{1}\left(z_{2}-z_{1}\right) \\
& -g_{1}\left[\left(z_{1 \tau}-z_{2 \tau}\right)-\left(z_{1}-z_{2}\right)\right] .
\end{aligned}
$$

The characteristic matrix of (11) is

$$
\lambda \mathbf{I}-\mathbf{C}-\mathbf{D} e^{-\lambda \tau}=\left(\begin{array}{cccc}
\lambda & -1 & 0 & 0 \\
-\omega_{1}^{\prime 2}-\alpha_{1}-g_{1}-g_{1} e^{-\lambda \tau} & \lambda+\xi_{1} & \alpha_{1}+g_{1}+g_{1} e^{-\lambda \tau} & 0 \\
0 & 0 & \lambda & -1 \\
\alpha_{2}+g_{2}+g_{2} e^{-\lambda \tau} & 0 & -\omega_{2}^{\prime 2}-\alpha_{2}-g_{2}-g_{2} e^{-\lambda \tau} & \lambda+\xi_{2}
\end{array}\right),
$$

where matrices $\mathbf{C}$ and $\mathbf{D}$ can be obtained by (11) as

$$
\begin{aligned}
& \mathbf{C}=\left[\begin{array}{cccc}
0 & 1 & 0 & 0 \\
-\omega_{1}^{\prime 2}-\alpha_{1}-g_{1} & -\xi_{1} & \alpha_{1}+g_{1} & 0 \\
0 & 0 & 0 & 1 \\
\alpha_{2}+g_{2} & 0 & -\omega_{2}^{\prime 2}-\alpha_{2}-g_{2} & -\xi_{2}
\end{array}\right], \\
& \mathbf{D}=\left[\begin{array}{cccc}
0 & 0 & 0 & 0 \\
g_{1} & 0 & -g_{1} & 0 \\
0 & 0 & 0 & 0 \\
-g_{2} & 0 & g_{2} & 0
\end{array}\right] .
\end{aligned}
$$

The characteristic equation can be obtained from (12) as

$$
\begin{aligned}
\lambda^{4} & +\lambda^{2}\left(g_{1}-g_{1} e^{-\lambda \tau}+g_{2}-g_{2} e^{-\lambda \tau}+\alpha_{1}+\alpha_{2}+\omega_{1}^{\prime 2}\right. \\
& \left.+\omega_{2}^{\prime 2}\right)+\omega_{1}^{\prime 2}\left(g_{2}-g_{2} e^{-\lambda \tau}\right)+\omega_{2}^{\prime 2}\left(g_{1}-g_{1} e^{-\lambda \tau}\right) \\
& +g_{1} \omega_{2}^{\prime 2}+g_{2} \omega_{1}^{\prime 2}+\omega_{1}^{\prime 2} \omega_{2}^{\prime 2}=0 .
\end{aligned}
$$

The imaginary part of eigenvalues is the natural frequency. Two eigenvalues of the characteristic equation (14) can be computed, whose imaginary parts are the smallest and real parts are the biggest, and then the first and second natural frequencies of the continuous structure can be obtained, which are shown in Figure 4.
From Figure 4, it can be seen that the second natural frequency decreases quickly as the time delay increases from 1.0 to 6.532 . When time delay reaches 6.532 , the first natural frequency can drop. The decrease of the first natural frequency makes it easier for the system to be resonant under low frequency periodic excitation. Therefore, the value of time delay should not be larger than 6.532 .

The real parts of eigenvalues determine the stability of the corresponding mode. The complex dynamics would be caused if there is more than one eigenvalue having positive real parts. The real parts of the eigenvalues corresponding to the first and second frequencies above can be obtained by solving (14) and thus shown in Figure 5.

In Figure 5, the dynamic behavior of the structure is periodic subject to external excitation when $\tau \in(0,1.162) \cup$ $(3.15,3.44)$ where no eigenvalues have positive real parts. The structure has complex dynamic behavior as $\tau \notin(0,1.162) \cup$ $(3.15,3.44)$ due to the appearance of eigenvalues with positive real parts.

From the discussions above, the nonlinear effects of the time delay on natural frequency and stability indicate that the time delay can greatly change the dynamic response of the structure through the variation of natural frequency and bifurcation behaviors induced. The detailed nonlinear mechanism and influence of the time delay on vibration suppression will be discussed further in the following section. 


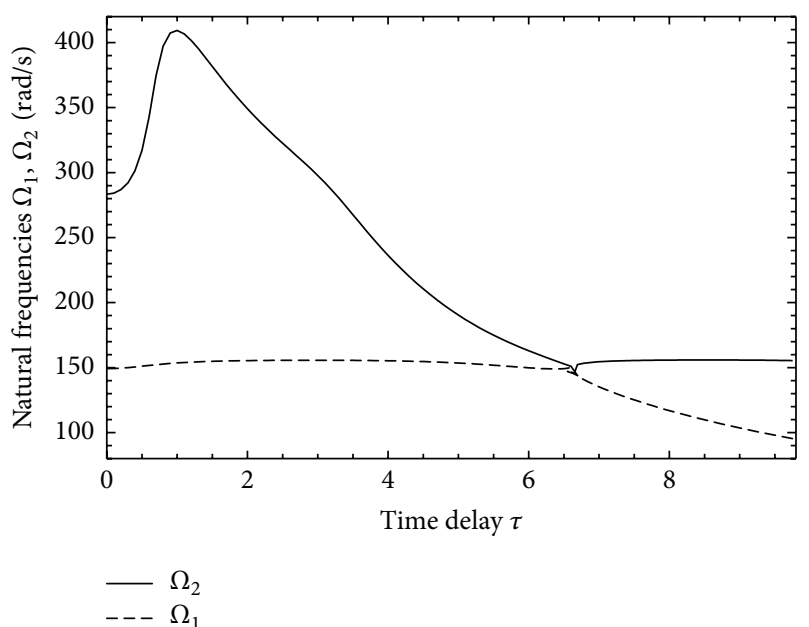

FIGURE 4: The relationship between the first and second natural frequency.

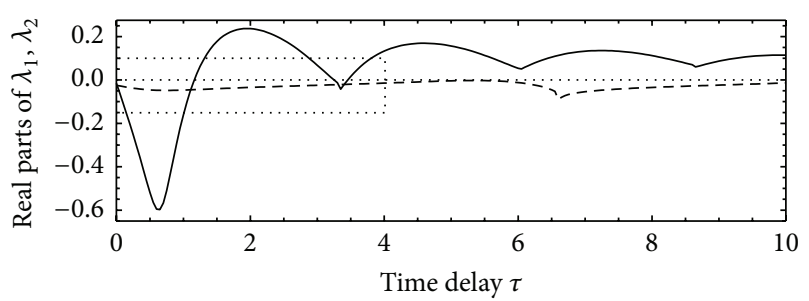

(a)

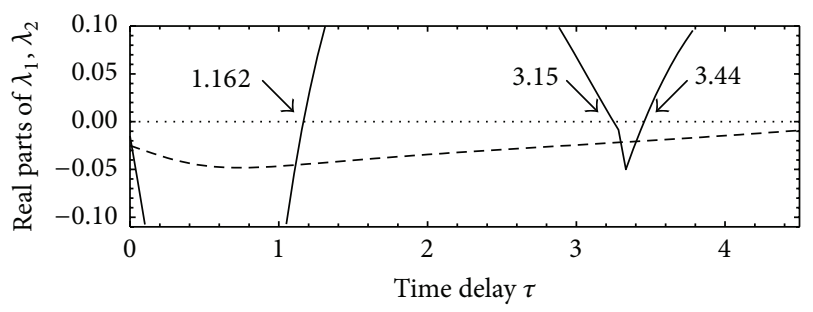

(b)

FIGURE 5: (a) is the real parts of eigenvalues under different time delay and (b) is the square region in (a).

\section{The Vibration Response of the Nonlinear Vibration Absorber with Time Delay}

4.1. Periodic Vibration Response with Time Delay. In this section, the output spectrum of the vibration response of the master structure is derived with the Harmonic Balance Method for the time delay $\tau \in(0,1.162) \cup(3.15,3.44)$. To this aim, it is assumed that the amplitudes caused by external excitations are as follows:

$$
\begin{aligned}
& z_{1}=a_{1}\left(t^{\prime}\right) \cos \left(\omega^{\prime} t^{\prime}\right)+a_{2}\left(t^{\prime}\right) \sin \left(\omega^{\prime} t^{\prime}\right) \\
& z_{2}=b_{1}\left(t^{\prime}\right) \cos \left(\omega^{\prime} t^{\prime}\right)+b_{2}\left(t^{\prime}\right) \sin \left(\omega^{\prime} t^{\prime}\right) .
\end{aligned}
$$

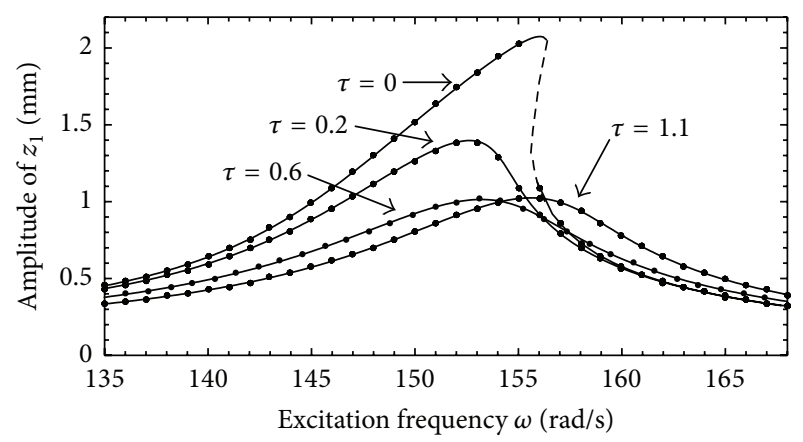

FIGURE 6: The response-frequency curves of the master structure when $\tau=0, \tau=0.2, \tau=0.6$, and $\tau=1$.1. Solid lines are solved by HBM while dots are solved by numerical simulations.

Substituting the expressions into (10) and finding the coefficients proportional to $\cos \left(\omega^{\prime} t^{\prime}\right)$ and $\sin \left(\omega^{\prime} t^{\prime}\right)$, the following nonlinear algebraic equations can be obtained:

$$
\begin{aligned}
& \left(g_{1}+\alpha_{1}+\omega_{1}^{\prime 2}-\omega^{\prime 2}-g_{1} \cos \tau \omega^{\prime}\right) a_{1} \\
& +\left(\xi_{1} \omega^{\prime}+g_{1} \sin \tau \omega^{\prime}\right) a_{2} \\
& -\left(g_{1}+\alpha_{1}+g_{1} \cos \tau \omega^{\prime}\right) b_{1}-g_{1} \sin \tau \omega^{\prime} b_{2}+\frac{3}{4} \gamma_{1} a_{1}^{3} \\
& +\frac{3}{4} \gamma_{1} a_{1} a_{2}^{2}+f=0 \\
& -\left(\xi_{1} \omega^{\prime}+g_{1} \sin \tau \omega^{\prime}\right) a_{1} \\
& +\left(g_{1}+\alpha_{1}-\omega^{\prime 2}+\omega_{1}^{\prime 2}-g_{1} \cos \tau \omega^{\prime}\right) a_{2} \\
& +g_{1} \sin \tau \omega^{\prime} b_{1}-\left(g_{1}+\alpha_{1}-g_{1} \cos \tau \omega^{\prime}\right) b_{2} \\
& +\frac{3}{4} \gamma_{1} a_{1}^{2} a_{2}+\frac{3}{4} \gamma_{1} a_{2}^{3}=0 \\
& \left(-g_{2}-\alpha_{2}+g_{2} \cos \tau \omega^{\prime}\right) a_{1}-a_{2} g_{2} \sin \tau \omega^{\prime} \\
& +\left(g_{2}+\alpha_{2}-\omega^{\prime 2}+\omega_{2}^{\prime 2}-g_{2} \cos \tau \omega^{\prime}\right) b_{1} \\
& +\left(\xi_{2} \omega^{\prime}+g_{2} \sin \tau \omega^{\prime}\right) b_{2}+\frac{3}{4} \gamma_{2} b_{1}^{3}+\frac{3}{4} \gamma_{2} b_{1} b_{2}^{2}=0 \\
& \left(\alpha_{2}+g_{2}-\omega^{\prime 2}+\omega_{2}^{\prime 2}-g_{2} \cos \tau \omega^{\prime}\right) b_{2}+g_{2} \sin \tau \omega^{\prime} a_{1} \\
& -\left(g_{2}+\alpha_{2}-g_{2} \cos \tau \omega^{\prime}\right) a_{2} \\
& -\left(\xi_{2} \omega^{\prime}+g_{2} \sin \tau \omega^{\prime}\right) b_{1}+\frac{3}{4} \gamma_{2} b_{1}^{2} b_{2}+\frac{3}{4} \gamma_{2} b_{2}^{3}=0
\end{aligned}
$$

The amplitude of the master structure is $A=\sqrt{a_{1}^{2}+a_{2}^{2}}$. Considering only the case when $\tau \in(0,1.162) \cup(3.15,3.44)$, Figure 6 presents the frequency response curves $A(\omega)$ under different values of the time delay.

Figure 6 presents the frequency response amplitude of the master structure around the resonance frequency $\omega=$ $154 \mathrm{rad} / \mathrm{s}$ under $\tau=0, \tau=0.2, \tau=0.6$, and $\tau=1.1$. The time 


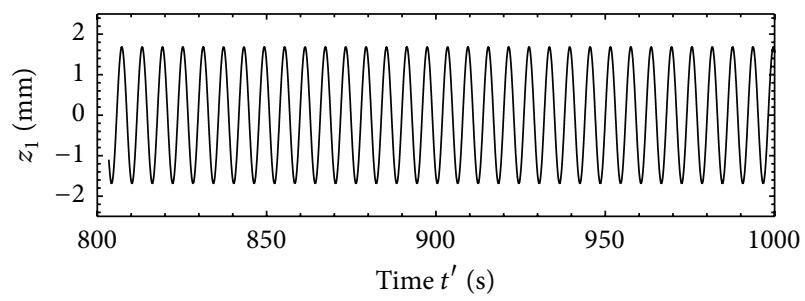

(a)

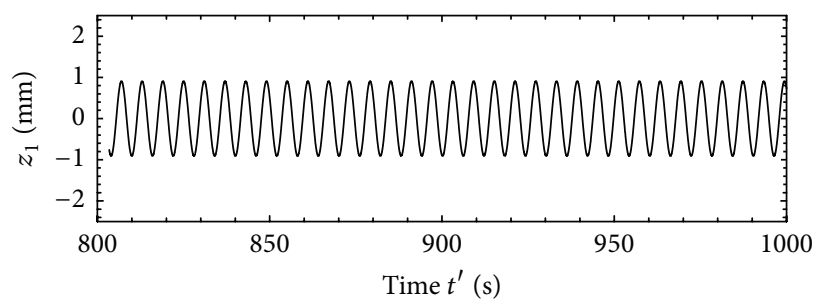

(b)

Figure 7: The time-series response when (a) $\tau=0$ and (b) $\tau=1.1$ as $\omega=154 \mathrm{rad} / \mathrm{s}$.

delay plays a significant role in vibration suppression when $\tau \in(0,1.162) \cup(3.15,3.44)$. It is noticed that, with the value of the time delay increasing, the peak response amplitude of the master structure decreases obviously. Also, the curve corresponding to $\tau=0$ jumps drastically when $\omega \approx 156 \mathrm{rad} / \mathrm{s}$, which shows that the cubic coefficients $\gamma_{1}$ and $\gamma_{2}$ affect the dynamic response of the structure. Figure 7 is the time-series response of the master structure for $\tau=0$ and $\tau=1.1$. It is shown that the vibration amplitude decreases by $50 \%$ with $\tau=1.1$ compared to that without time delay as $\omega=154 \mathrm{rad} / \mathrm{s}$. The mechanism of the vibration absorber with time delay is that the introduced time delay adjusts the output response of the servo motor so that the active force acting on the master structure could counteract the original motion.

4.2. Coupled Vibration Modes. It has been mentioned that the system characteristic equation will have eigenvalues with positive real parts for $\tau \in(1.162,3.15)$. The bifurcation behavior would be incurred by the time delay, which can be coupled with the nonlinear motion of the system induced by external excitation. When the natural frequency of the master structure is designed around half of resonance frequency of the slave structure, vibration energy transmission could happen between these coupled modes.

4.2.1. Derivation of the Solutions of Vibration Modes. To investigate the coupling vibration modes, the vibration solution can be derived, which can be expressed in two parts including motions induced by the time delay and external excitation, respectively. The frequency of the first part in the solution should equal the input frequency of external excitation while the frequency of the second part should equal the frequency at bifurcation point. From (14), the critical time delay for producing the bifurcation motion is $\tau_{c}=3.15$ and the dimensionless frequency of bifurcation mode is $\Omega_{c}^{\prime}=$ 1.9237. The solution for $\tau \in(1.162,3.15)$ can be expressed as follows:

$$
\mathbf{Z}=\left(\begin{array}{c}
z_{1} \\
y_{1} \\
z_{2} \\
y_{2}
\end{array}\right)=\mathbf{Z}_{1}+\mathbf{Z}_{2}=\left(\begin{array}{l}
z_{11} \\
y_{11} \\
z_{21} \\
y_{21}
\end{array}\right)+\left(\begin{array}{l}
z_{12} \\
y_{12} \\
z_{22} \\
y_{22}
\end{array}\right),
$$

where $z_{i 1}$ is the motion by the external excitation and $z_{i 2}$ is the bifurcation motion caused by the time delay. As mentioned in (17), the frequency of $z_{i 1}$ equals the input frequency of external excitation, and the frequency of $z_{i 2}$ equals the frequency at bifurcation point.

The two parts of the solution are difficult to be derived because the two modes are coupled by nonlinear terms. But the linear part of the vibration equations can be separated to find the eigenvector of each mode. Substituting the expression of solutions (17) into (10), the linear part can be separated from the equations. The expression of the motion excited by the external excitation can therefore be simplified.

Two time scales are introduced and given by $t^{\prime}=T_{0}$ and $\varepsilon t^{\prime}=T_{1}$; then one has

$$
\begin{aligned}
\frac{d \mathbf{Z}}{d t^{\prime}}= & \frac{\partial \mathbf{Z}}{\partial T_{0}}+\varepsilon \frac{\partial \mathbf{Z}}{\partial T_{1}}, \\
\mathbf{Z}_{1}= & \mathbf{Z}_{10}\left(T_{0}, T_{1}\right)+\varepsilon \mathbf{Z}_{11}\left(T_{0}, T_{1}\right), \\
\mathbf{Z}_{2}= & \mathbf{Z}_{20}\left(T_{0}, T_{1}\right)+\varepsilon \mathbf{Z}_{21}\left(T_{0}, T_{1}\right), \\
\mathbf{Z}_{1}\left(t^{\prime}-\tau\right)= & \mathbf{Z}_{1}\left(T_{0}-\tau, T_{1}-\varepsilon \tau\right) \\
= & \mathbf{Z}_{10}\left(T_{0}-\tau, T_{1}-\varepsilon \tau\right) \\
& +\varepsilon \mathbf{Z}_{11}\left(T_{0}-\tau, T_{1}-\varepsilon \tau\right), \\
\mathbf{Z}_{2}\left(t^{\prime}-\tau\right)= & \mathbf{Z}_{2}\left(T_{0}-\tau, T_{1}-\varepsilon \tau\right) \\
= & \mathbf{Z}_{20}\left(T_{0}-\tau, T_{1}-\varepsilon \tau\right) \\
& +\varepsilon \mathbf{Z}_{21}\left(T_{0}-\tau, T_{1}-\varepsilon \tau\right) .
\end{aligned}
$$

Perturbing $\tau$ in $\varepsilon \tau_{\varepsilon}$ as

$$
\tau=\tau_{c}+\varepsilon \tau_{\varepsilon}
$$

and expanding the vector $\mathbf{Z}\left(t^{\prime}\right)$, the time-delay items $z_{i}\left(t^{\prime}-\tau\right)$ are as follows:

$$
\begin{aligned}
z_{1}\left(t^{\prime}-\tau\right)= & z_{110}\left(T_{0}-\tau_{c}, T_{1}\right)+\varepsilon z_{111}\left(T_{0}-\tau_{c}, T_{1}\right) \\
& -\varepsilon \tau_{\varepsilon} \frac{\partial}{\partial T_{1}} z_{110}\left(T_{0}-\tau_{c}, T_{1}\right) \\
& +z_{120}\left(T_{0}-\tau_{c}, T_{1}\right)
\end{aligned}
$$




$$
\begin{aligned}
& +\varepsilon z_{121}\left(T_{0}-\tau_{c}, T_{1}\right) \\
& -\varepsilon \tau_{\varepsilon} \frac{\partial}{\partial T_{1}} z_{120}\left(T_{0}-\tau_{c}, T_{1}\right)+o(\varepsilon) \\
z_{2}\left(t^{\prime}-\tau\right)= & z_{210}\left(T_{0}-\tau_{c}, T_{1}\right)+\varepsilon z_{211}\left(T_{0}-\tau_{c}, T_{1}\right) \\
& -\varepsilon \tau_{\varepsilon} \frac{\partial}{\partial T_{1}} z_{210}\left(T_{0}-\tau_{c}, T_{1}\right) \\
& +z_{220}\left(T_{0}-\tau_{c}, T_{1}\right) \\
& +\varepsilon z_{221}\left(T_{0}-\tau_{c}, T_{1}\right) \\
& -\varepsilon \tau_{\varepsilon} \frac{\partial}{\partial T_{1}} z_{220}\left(T_{0}-\tau_{c}, T_{1}\right)+o(\varepsilon)
\end{aligned}
$$

The first subscript is the number of degrees of freedom, the second is the number of modes, and the third is the order of perturbation. Substituting (18)-(20) into the dynamical equation (10), one has

$$
\begin{aligned}
& \frac{d}{d T_{0}}\left(z_{110}+z_{120}\right)=\left(y_{110}+y_{120}\right) \\
& \frac{d}{d T_{0}}\left(y_{110}+y_{120}\right)=-\omega_{1}^{\prime 2}\left(z_{110}+z_{120}\right)-\xi_{1}\left(y_{110}\right. \\
& \left.+y_{120}\right)+\alpha_{1}\left[\left(z_{210}+z_{220}\right)-\left(z_{110}+z_{120}\right)\right] \\
& +g_{1}\left[\left(z_{110 \tau_{c}}+z_{120 \tau_{c}}-z_{210 \tau_{c}}-z_{220 \tau_{c}}\right)\right. \\
& \left.-\left(z_{110}+z_{120}-z_{210}-z_{220}\right)\right] \text {, } \\
& \frac{d}{d T_{0}}\left(z_{210}+z_{220}\right)=\left(y_{210}+y_{220}\right) \text {, } \\
& \frac{d}{d T_{0}}\left(y_{210}+y_{220}\right)=-\omega_{2}^{\prime 2}\left(z_{210}+z_{220}\right)-\xi_{2}\left(y_{210}\right. \\
& \left.+y_{220}\right)+\alpha_{2}\left[\left(z_{110}+z_{120}\right)-\left(z_{210}+z_{220}\right)\right] \\
& +g_{2}\left[\left(z_{210 \tau_{c}}+z_{220 \tau_{c}}-z_{110 \tau_{c}}-z_{120 \tau_{c}}\right)\right. \\
& \left.-\left(z_{210}+z_{220}-z_{110}-z_{120}\right)\right] \text {, } \\
& \frac{d}{d T_{0}}\left(z_{111}+z_{121}\right)=\left(y_{111}+y_{121}\right)-\frac{d}{d T_{0}}\left(z_{110}+z_{120}\right) \text {, } \\
& \frac{d}{d T_{0}}\left(y_{111}+y_{121}\right)=-\frac{d}{d T_{0}}\left(y_{110}+y_{120}\right)-\omega_{1}^{\prime 2}\left(z_{111}\right. \\
& \left.+z_{121}\right)-\xi_{1}\left(y_{111}+y_{121}\right)+\frac{f}{2}\left(e^{-i \omega^{\prime} T_{0}}+\mathrm{cc} .\right) \\
& +\alpha_{1}\left[\left(z_{211}+z_{221}\right)-\left(z_{111}+z_{121}\right)\right] \\
& +g_{1}\left[\left(z_{111 \tau_{c}}+z_{121 \tau_{c}}-z_{211 \tau_{c}}-z_{221 \tau_{c}}\right)\right. \\
& \left.-\left(z_{111}+z_{121}-z_{211}-z_{221}\right)\right]+\gamma_{1}\left(z_{110}^{3}+z_{120}^{3}\right.
\end{aligned}
$$

$$
\begin{aligned}
& \left.\quad+3 z_{120}^{2} z_{110}+3 z_{110}^{2} z_{120}\right)+g_{1} \tau_{\varepsilon} \frac{d}{d T_{1}}\left(z_{110 \tau_{c}}\right. \\
& \left.+z_{120 \tau_{c}}-z_{210 \tau_{c}}-z_{220 \tau_{c}}\right) \\
& \frac{d}{d T_{0}}\left(z_{211}+z_{221}\right)=\left(y_{211}+y_{221}\right)-\frac{d}{d T_{0}}\left(z_{210}+z_{220}\right) \\
& \frac{d}{d T_{0}}\left(y_{211}+y_{221}\right)=-\frac{d}{d T_{0}}\left(y_{210}+y_{220}\right)-\xi_{2}\left(y_{211}\right. \\
& \left.\quad+y_{221}\right)-g_{2} \tau_{\varepsilon} \frac{d}{d T_{1}}\left(z_{210 \tau_{c}}+z_{220 \tau_{c}}-z_{210 \tau_{c}}\right. \\
& \left.\quad-z_{220 \tau_{c}}\right)-\omega_{2}^{\prime 2}\left(z_{211}+z_{221}\right) \\
& -g_{2}\left[\left(z_{211 \tau_{c}}+z_{221 \tau_{c}}-z_{111 \tau_{c}}-z_{121 \tau_{c}}\right)\right. \\
& \left.-\left(z_{211}+z_{221}-z_{111}-z_{121}\right)\right]+\alpha_{2}\left[\left(z_{111}+z_{121}\right)\right. \\
& \left.-\left(z_{211}+z_{221}\right)\right]+\gamma_{2}\left(z_{210}^{3}+z_{220}^{3}+3 z_{220}^{2} z_{210}\right. \\
& \left.+3 z_{210}^{2} z_{220}\right) .
\end{aligned}
$$

The frequency of the external excitation $\omega^{\prime}$ is near the natural frequency of the structure $\Omega_{1}^{\prime}$ while the bifurcation frequency $\Omega_{c}^{\prime}$ is close to $2 \Omega_{1}^{\prime}$. Tuning parameters $\sigma_{1}$ and $\sigma_{2}$ are introduced to describe the difference between $\omega^{\prime}$ and $\Omega_{1}^{\prime}$ and $\Omega_{c}^{\prime}$ and $2 \Omega_{1}^{\prime}$, respectively. The following expressions for $\omega^{\prime}$ and $\Omega_{c}^{\prime}$ are given:

$$
\begin{aligned}
& \omega^{\prime}=\Omega_{1}^{\prime}+\varepsilon \sigma_{1}, \\
& \Omega_{c}^{\prime}=2 \Omega_{1}^{\prime}+\varepsilon \sigma_{2} .
\end{aligned}
$$

For $\tau=\tau_{c}$, the nontrivial solution to (10) can be written as

$$
\begin{aligned}
& \mathbf{Z}_{10}+\mathbf{Z}_{20}=\left(\begin{array}{c}
z_{110} \\
y_{110} \\
z_{210} \\
y_{210}
\end{array}\right)+\left(\begin{array}{c}
z_{120} \\
y_{120} \\
z_{220} \\
y_{220}
\end{array}\right) \\
& =A\left(T_{1}\right) e^{i \Omega_{1}^{\prime} T_{0}}\left(\begin{array}{c}
1 \\
v_{12} \\
v_{13} \\
v_{14}
\end{array}\right) \\
& +B\left(T_{1}\right) e^{i \Omega_{c}^{\prime} T_{0}}\left(\begin{array}{c}
1 \\
v_{22} \\
v_{23} \\
v_{24}
\end{array}\right)+c c .,
\end{aligned}
$$


where cc. stands for the complex conjugation of the preceding terms and the eigenvector $\left(\begin{array}{llll}1 & v_{12} & v_{13} & v_{14}\end{array}\right)^{T}$ is determined by

$$
\left(i \Omega_{1}^{\prime} \mathbf{I}-\mathbf{C}-\mathbf{D} e^{-i \Omega_{1}^{\prime} \tau}\right)\left(\begin{array}{c}
1 \\
v_{12} \\
v_{13} \\
v_{14}
\end{array}\right)=0
$$

and $\left(\begin{array}{llll}1 & v_{22} & v_{23} & v_{24}\end{array}\right)^{T}$ is determined by

$$
\left(i \Omega_{c}^{\prime} \mathbf{I}-\mathbf{C}-\mathbf{D} e^{-i \Omega_{c}^{\prime} \tau_{c}}\right)\left(\begin{array}{c}
1 \\
v_{22} \\
v_{23} \\
v_{24}
\end{array}\right)=0 .
$$

To obtain the amplitude and frequency of the motion, $A\left(T_{1}\right)$ and $B\left(T_{1}\right)$ are set as

$$
\begin{aligned}
& A\left(T_{1}\right)=\frac{1}{2} r_{1}\left(T_{1}\right) \exp \left(i \beta_{1}\left(T_{1}\right)\right), \\
& B\left(T_{1}\right)=\frac{1}{2} r_{2}\left(T_{1}\right) \exp \left(i \beta_{2}\left(T_{1}\right)\right) .
\end{aligned}
$$

Substituting (27) into the coefficient of $\varepsilon$ in (22) and separating the real and imaginary part of secular terms, the normal form can be obtained as

$$
\begin{aligned}
\dot{r}_{1}= & \mu_{1}\left(\Omega_{1}^{\prime}, \sigma_{1}, \tau, \tau_{\varepsilon}, f^{\prime}\right) r_{1}+a_{11}\left(\Omega_{1}^{\prime}, \sigma_{1}, \tau, f^{\prime}\right) r_{1}^{3} \\
& +a_{12}\left(\Omega_{1}^{\prime}, \sigma_{1}, \tau, f^{\prime}\right) r_{1} r_{2}^{2}, \\
\dot{r}_{2}= & \mu_{2}\left(\Omega_{1}^{\prime}, \sigma_{2}, \tau, \tau_{\varepsilon}, f^{\prime}\right) r_{2}+a_{21}\left(\Omega_{1}^{\prime}, \sigma_{2}, \tau, f^{\prime}\right) r_{2}^{3} \\
& +a_{22}\left(\Omega_{1}^{\prime}, \sigma_{2}, \tau, f^{\prime}\right) r_{2} r_{1}^{2}, \\
\dot{\beta}_{1}= & b_{13}\left(\Omega_{1}^{\prime}, \sigma_{1}, \sigma_{2}, \tau, \tau_{\varepsilon}, f^{\prime}\right)+b_{11}\left(\Omega_{1}^{\prime}, \tau, f^{\prime}\right) r_{1}^{2} \\
& +b_{12}\left(\Omega_{1}^{\prime}, \tau, f^{\prime}\right) r_{2}^{2}, \\
\dot{\beta}_{2}= & b_{23}\left(\Omega_{1}^{\prime}, \sigma_{1}, \sigma_{2}, \tau, \tau_{\varepsilon}, f^{\prime}\right)+b_{21}\left(\Omega_{1}^{\prime}, \tau, f^{\prime}\right) r_{1}^{2} \\
& +b_{22}\left(\Omega_{1}^{\prime}, \tau, f^{\prime}\right) r_{2}^{2} .
\end{aligned}
$$

After substituting the values of parameters, the critical value of time delay $\tau_{c 2}=3.15$ and $\tau_{\varepsilon}=\tau-\tau_{c 2}$, into the equations above, the normal form of (28) is given in (A.1). The solution of the two vibration modes can therefore be obtained by solving $\dot{r}_{1}=0, \dot{r}_{2}=0, \dot{\beta}_{1}=0$, and $\dot{\beta}_{2}=0$.

4.2.2. The Bifurcation Modes and Critical Points. Figure 8 shows the vibration amplitude of the master structure as the time delay varies. There are three bifurcation points in Figure 8(b), which are $H_{1}, H_{2}$, and $H_{3}$. The resonance behavior among different modes and the transmission of energy are different at $H_{1}, H_{2}$, and $H_{3}$ due to the fact that the critical time delay $\tau_{c}$ and bifurcation frequency $\Omega_{c}^{\prime}$ at these three bifurcation points are different. The amplitude drops continuously when $\tau \in(0,1.162)$ and jumps from $1 \mathrm{~mm}$ to nearly $0.5 \mathrm{~mm}$ at $H_{1}$ where $\tau_{c 1}=1.162$. The amplitude curves are discontinuous at $H_{1}$, which means that the bifurcation undergoes a subcritical Hopf bifurcation induced by the time delay at point $H_{1}$ and makes the amplitude of the master structure jump.

As time delay $\tau \in(1.162,3.15)$, the vibration amplitude keeps at a low level. The time delay is helpful for vibration suppression in this range. The second critical value of the time delay for bifurcation is $H_{2}$ where $\tau_{c 2}=3.15$. The frequency of bifurcation mode is $\Omega_{c 2}^{\prime}=1.924$ and is close to two times the first natural frequency. The amplitude of the master structure increases continuously for the occurrence of supercritical bifurcation at point $\mathrm{H}_{2}$ where the bifurcation mode and excitation mode are in weak resonance. The weak resonance helps the vibration suppression in that the bifurcation mode absorbs the vibration energy of the master structure. The vibration energy is transferred from $r_{1}$ to $r_{2}$ because of mode coupling by the nonlinearity of the system. The slave structure absorbs the vibration energy from the master structure.

When the time delay reaches the value $\tau_{c 3}=3.44$, the third Hopf bifurcation $\mathrm{H}_{3}$ occurs. From Figure 5, an eigenvalue crosses the real axis, which results in a subcritical Hopf bifurcation. The amplitude of the master structure jumps to a large value there in Figure 8. At this bifurcation point, the frequency of the bifurcation mode is $\Omega_{c 3}^{\prime}=2.74$, which is close to three times the first natural frequency $\Omega_{1}^{\prime}$ as $\Omega_{c 3}^{\prime} \approx 3 \Omega_{1}^{\prime}$. The two modes are in strong resonance when time delay $\tau>\tau_{c 3}=3.44$. The strong resonance cannot help the vibration absorbing because of the extra energy imported.

Figure 9 can clearly show the ratio of the frequencies of the motion at around the bifurcation points $\mathrm{H}_{2}$ and $\mathrm{H}_{3}$. The frequency spectrum of the dynamic response of the master structure contains two frequencies subject to a single-tone excitation. The ratio of the frequencies of bifurcation mode and the first mode is $1 / 2$ at around point $H_{2}$ while it is $1 / 3$ at around $\mathrm{H}_{3}$. Due to the magnitude level, the former mode can be regarded as being in weak resonances while the latter can be regarded as being in strong resonance.

4.2.3. Vibration Performance in Different Bifurcation Modes. Figure 10 shows the dynamic behavior of the master structure as $\tau=2$. Figure 10(a) is the time-series response of the master structure; Figure 10(b) is the phase diagram and Figure 10 (c) is the Poincare mapping section. There is a cycle on the Poincare mapping, which indicates that the dynamic behavior of the system is quasi-periodic motion including two irreducible frequencies. As the bifurcation mode and excitation mode are in $1 / 2$ weak resonance, the amplitude of the master structure can be controlled in a small range. The vibration suppression performance of the nonlinear vibration absorber is significant for $\tau \in(1.162,3.15)$ owing to the fact that the energy could be transferred from the excitation mode to the bifurcation mode.

Figure 11 shows the motion of the master structure as $\tau=3.5$. The system undergoes a subcritical bifurcation at $H_{3}$ where $\tau_{c 3}=3.44$. From Figure 11, the dynamics of the 


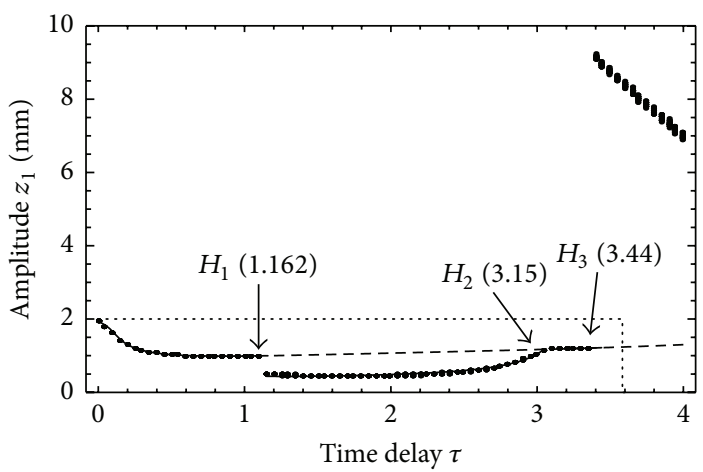

(a)

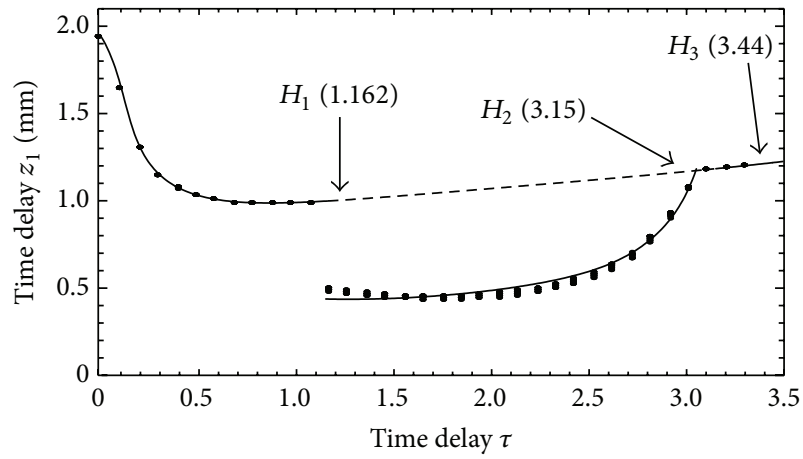

(b)

Figure 8: (a) is the amplitude of the master structure under different time delay as the frequency of excitation $\omega=154 \mathrm{rad} / \mathrm{s}$ and (b) is the square region in (a). For $\tau \in(0,1.162) \cup(3.15,3.44)$, the solid line is stability periodic motion by analysis via Harmonic Balance Method (HBM), and for $\tau \in(1.162,3.15)$, the solid line is obtained via Multiscale Method (MSM). The dashed line is unstable motion obtained by $\mathrm{HBM}$ and the dots are the results by numerical simulation.

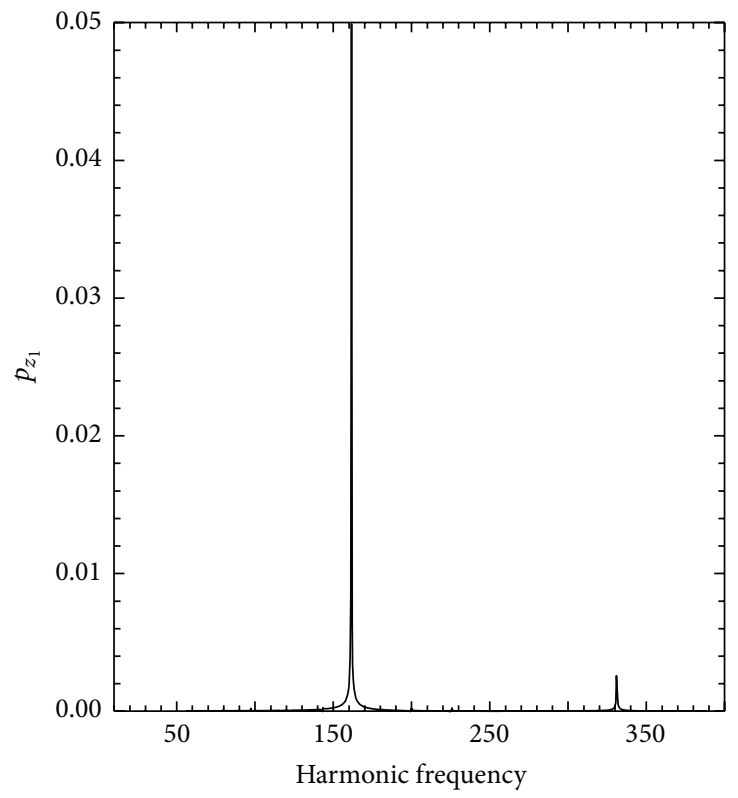

(a)

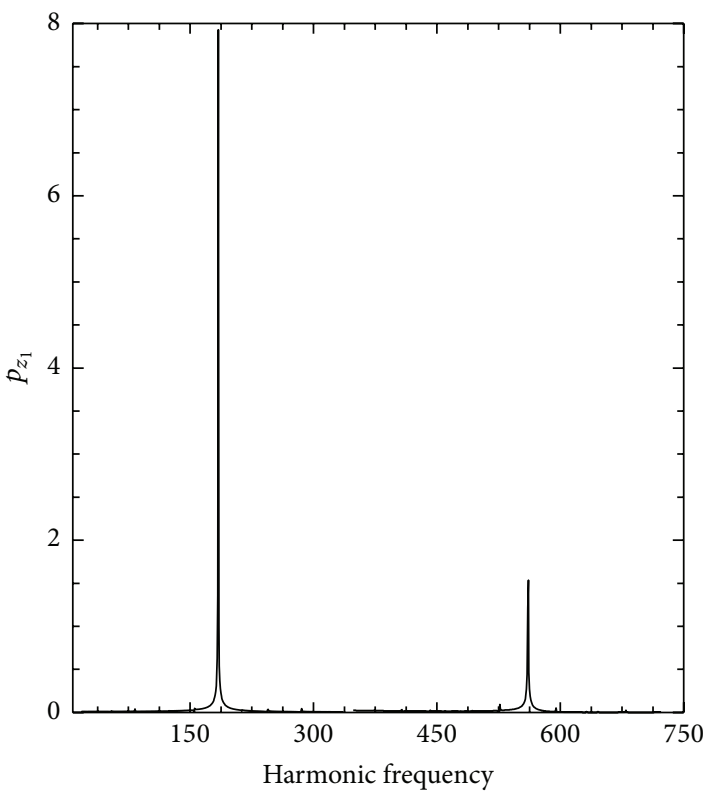

(b)

FIgURE 9: The frequency spectrum for (a) $\tau=3.05$ and (b) $\tau=3.46$.

structure contain two modes and when $\tau>\tau_{c 3}$ the vibration amplitude of the master structure is much larger than that when $\tau<\tau_{c 3}$. The ratio of the bifurcation mode frequency and the first natural frequency is about $1 / 3$ here; thus the two modes incurred by bifurcation and excitation are in strong resonance. The amplitude of the master structure jumps to $10 \mathrm{~mm}$ at $\mathrm{H}_{3}$ for the strong energy transmission between the two modes. The amplitude of the master structure cannot be suppressed because the time delay cannot dissipate vibration energy in this case. Therefore, different energy transmission mechanism in weak and strong resonance results in very different effect in vibration suppression.

4.2.4. Energy Transfer Phenomena between Two Vibration Modes. It has been shown that the motion bifurcated by the time delay is helpful to vibration suppression for $\tau \in(1.162,3.15)$. The vibration absorber effect for $\tau \in$ $(1.162,3.15)$ is more significant than that in other ranges because the vibration absorber may utilize the energy transmission of coupling modes to realize the active vibration 


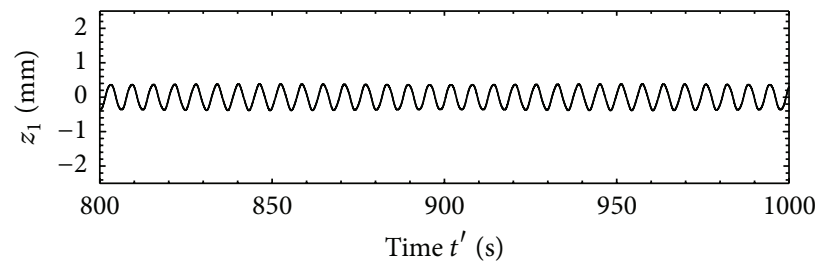

(a)

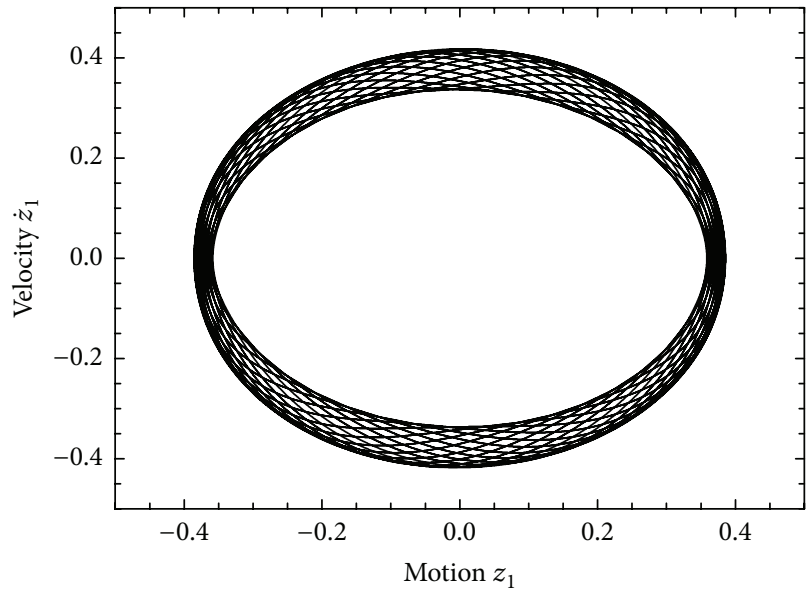

(b)

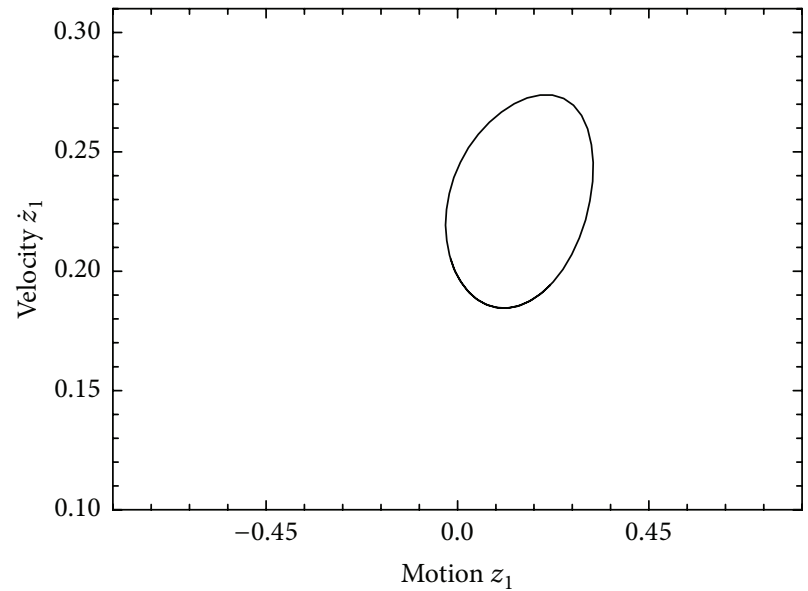

(c)

Figure 10: The time series and phase diagram when $\tau=2$ and $\omega=154 \mathrm{rad} / \mathrm{s}$ : (a) time series; (b) the phase diagram; (c) the Poincare mapping section.

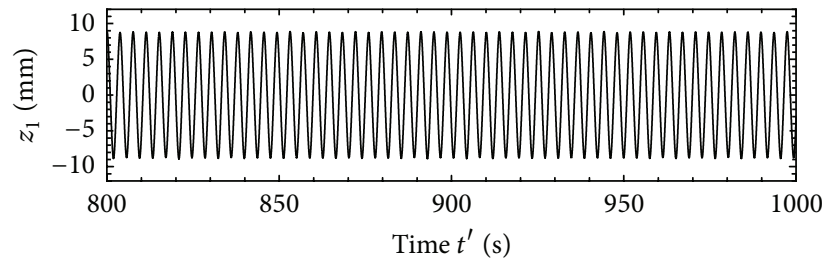

(a)

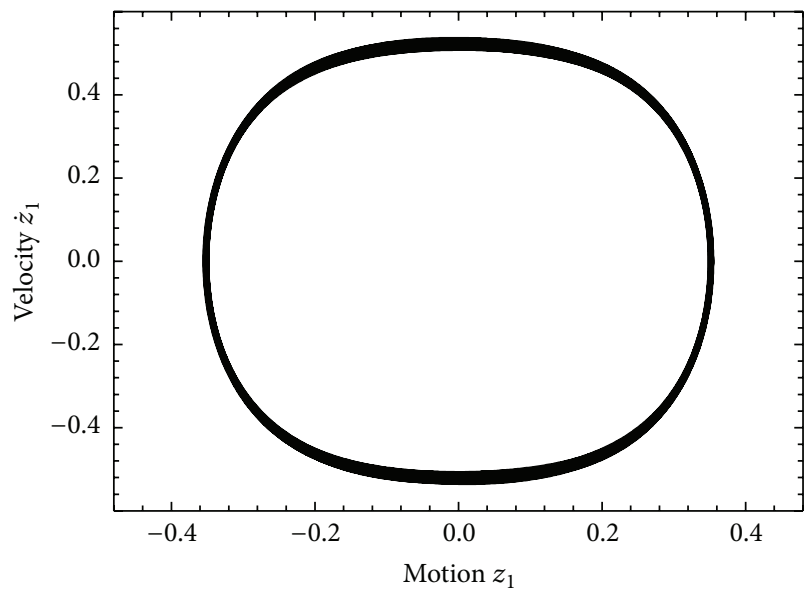

(b)

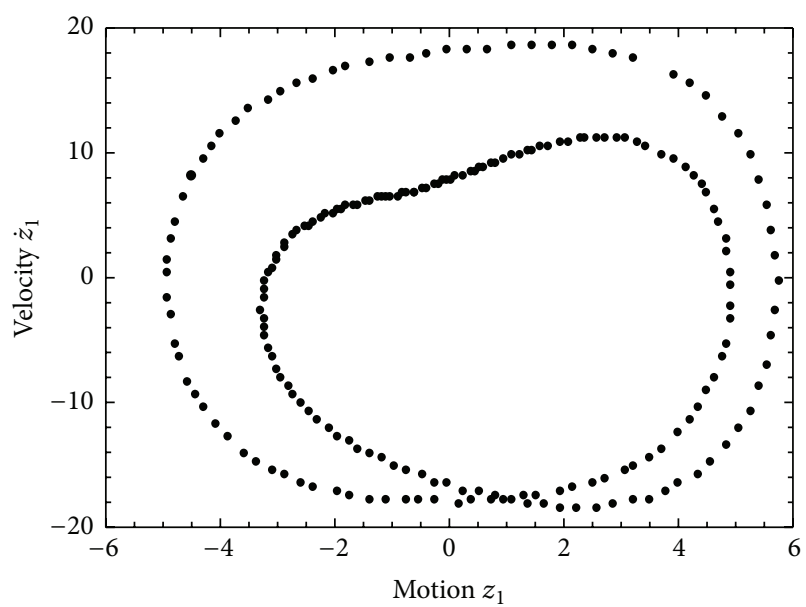

(c)

Figure 11: The time series and Poincare mapping section for $\tau=3.5$ and $\omega=154 \mathrm{rad} / \mathrm{s}$ : (a) time series; (b) the phase diagram; (c) the Poincare mapping section. 


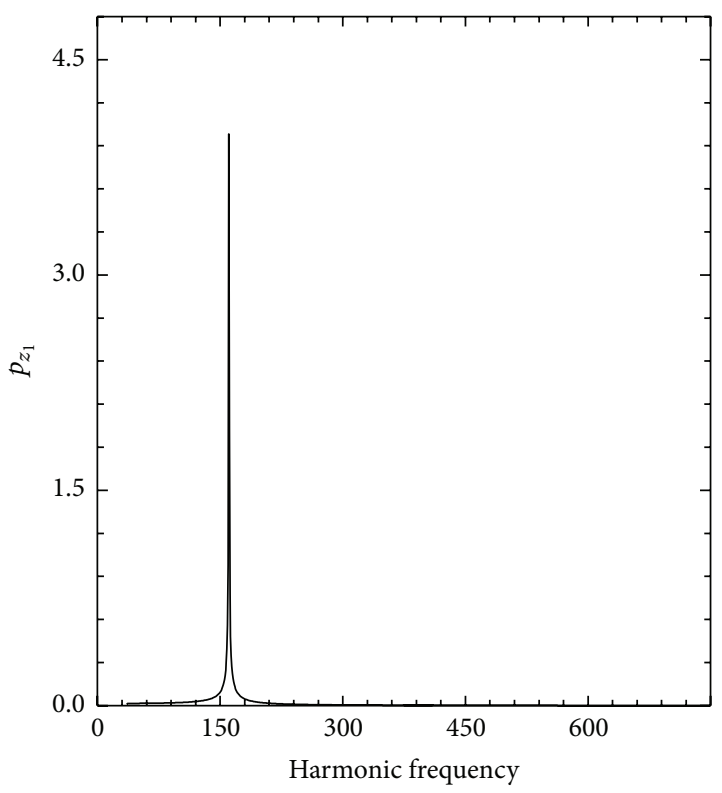

(a)

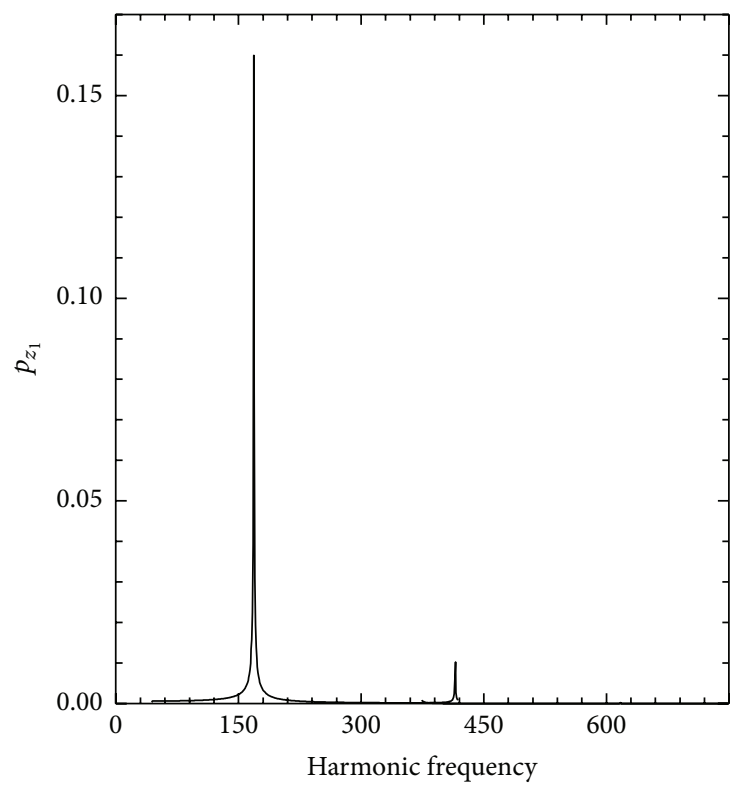

(c)

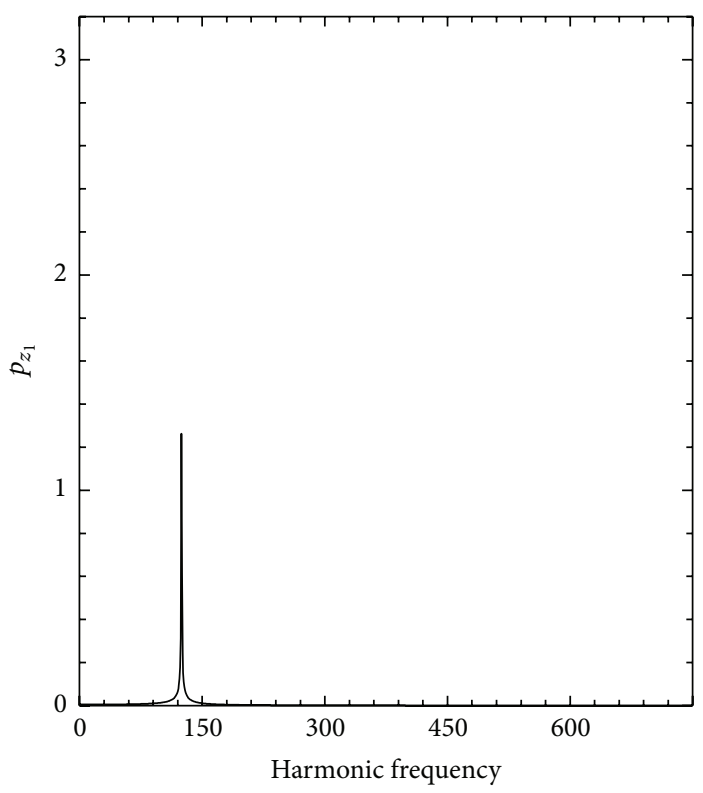

(b)

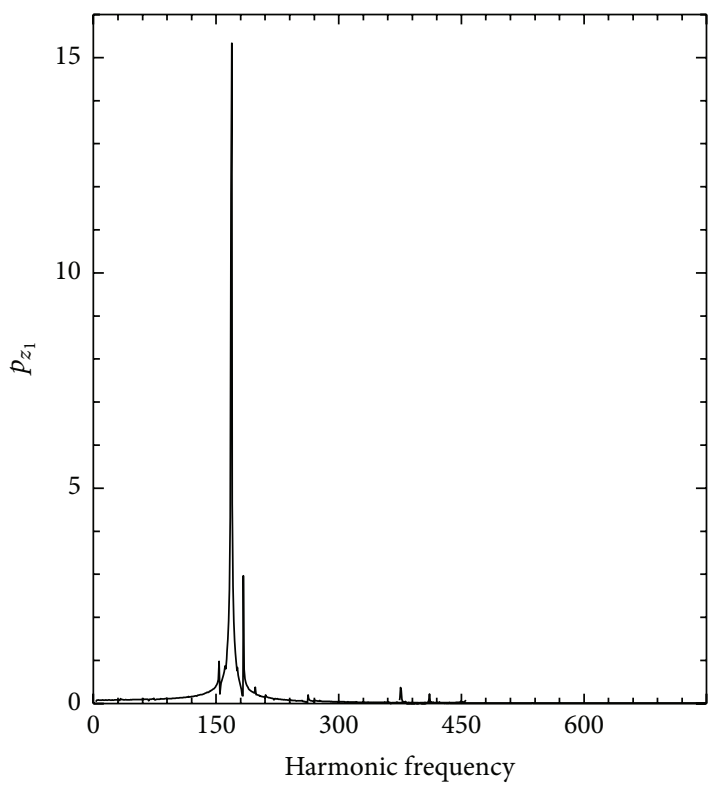

(d)

FIgURE 12: The frequency spectrum for (a) $\tau=0$, (b) $\tau=1.1$, (c) $\tau=2$, and (d) $\tau=5$.

absorbing. The mode bifurcated by the time delay could absorb the vibration energy of external excitation.

To demonstrate this more clearly, Figure 12 indicates the energy distribution of the vibration response under different time delays. When $\tau=0$, the vibration of the master structure contains huge energy at the low frequency. The frequency spectrum for $\tau=1.1$ shows that the vibration energy diminishes with the time delay increasing while it keeps the same frequency as $\tau=0$. When the time delay increases to $\tau=$ 2 , the vibration energy at the first frequency reduces several times lower than that when $\tau<\tau_{c 1}=1.162$. Also, there is vibration energy at the second frequency, which implies that the bifurcation induced by the time delay transfers some energy from a low frequency to a high one. When $\tau=5$, the dynamic response of the system contains more than two frequencies and the vibration energy is higher than other cases. It can be seen that the phenomena observed here are consistent with these by the stability analysis in Section 3 .

The most significant region of the time delay affecting vibration absorbing is $\tau \in(1.162,3.15)$. The value of the time 
delay $\tau$ in the analysis above is a dimensionless parameter. After carrying out an inverse procedure of dimensional transformation, the value of time delay $\tau_{k}$ in practical engineering can be obtained by $\tau_{k}=\tau / \Omega^{\prime}=\tau \sqrt{m_{1} / k_{1}}=0.006786 \tau(\mathrm{s})$. As a result, the best range of time delay in practical engineering is that $\tau_{k} \in(0.00788537,0.022055)(\mathrm{s})$, which can be realized in practice.

\section{Conclusions}

In this study, the time-delayed active control of vibration absorbers coupled with a continuous beam structure is studied. The vibration amplitude of the master structure decreases nearly by $50 \%$ when the time delay falls into a specific range; that is, $\tau \in(0,1.162) \cup(3.15,3.44)$. The timedelayed vibration absorber engenders opposite motion on the master structure, which can counteract part of its vibration energy. Moreover, it is shown that different values of time delay have different effects on vibration absorbing behavior of the structure. The bifurcation mode induced by the time delay helps the vibration absorbing for $\tau \in(1.162,3.15)$. The mode bifurcated by the time delay and the first mode of the system are in 1/2 weak resonance, which can transfer some vibration energy out of the mode at low frequency and therefore decrease the amplitude of the master structure at corresponding frequency range. However, at $\tau=3.44$, the $1 / 3$ strong resonance makes the amplitude of the master structure enlarged because the bifurcation caused by the time delay amplifies vibration energy of the system. The results obtained in this study could provide a significant insight into the analysis and design of active vibration absorbers with deliberately introduced time delay by exploiting nonlinear benefits for realizing superior vibration suppression.

\section{Appendix}

The normal form at around $H_{2}$ for (28) is given by

$$
\begin{aligned}
& 1.176 r_{1}+22500 r_{1}^{3}+45000 r_{1} r_{2}^{2}-3.66794 \\
& \quad \times 10^{6} r_{1}^{2} r_{2} \sigma_{2}-1.03 r_{1}-2.03 r_{1} \sigma_{1}+f^{\prime} \\
& \quad-0.126657 r_{1} \cos \left(1+\sigma_{1}\right) \tau \\
& \quad+1.6034 r_{1} \sin \left(1+\sigma_{1}\right) \tau=0 \\
& 17.15408 r_{1}+411868 r_{1}^{3}+6.6415 \times 10^{9} r_{1} r_{2}^{2} \\
& \quad-3.66794 \times 10^{7} r_{1}^{2} r_{2} \sigma_{2}-r_{1}\left(1+2 \sigma_{1}\right) \\
& \quad-1.544 r_{1} \sin \left(1+\sigma_{1}\right) \tau \\
& \quad+15.034 r_{1} \cos \left(1+\sigma_{1}\right) \tau=0 \\
& 6022.53 r_{2}-1.2538 \times 10^{8} r_{1}^{2} r_{2}-5.05449 \times 10^{11} r_{2}^{3} \\
& \quad+552.872 r_{2} \sigma_{1}-1882.04 r_{2} \tau=0,
\end{aligned}
$$

$$
\begin{aligned}
& -1900.07 r_{2}-4.40917 \times 10^{8} r_{1}^{2} r_{2}-1.77748 \times 10^{12} r_{2}^{3} \\
& +3072.32 r_{2} \sigma_{1}+593.772 r_{2} \tau=0 .
\end{aligned}
$$

\section{Physical Parameters of the Structure}

$l_{i}: \quad$ Length of each beam $(\mathrm{m})$

$C_{i}$ : Damping coefficient $\left(\mathrm{N} \cdot \mathrm{s} \cdot \mathrm{m}^{-1}\right)$

$k_{3}$ : Stiffness of coupling spring $\left(\mathrm{N} \cdot \mathrm{m}^{-1}\right)$

$k_{\alpha}$ : Stiffness of control spring $\left(\mathrm{N} \cdot \mathrm{m}^{-1}\right)$

E: Elastic modulus of the material, $2.06 \times 10^{11}(\mathrm{~Pa})$

$I_{i}:$ Moment of inertia $\left(\mathrm{m}^{4}\right)$

$\omega$ : Frequency of external force $\left(\mathrm{rad} \cdot \mathrm{s}^{-1}\right)$

$F$ : Amplitude of external force (N)

$w_{i}$ : Deflection of each beam $(\mathrm{m})$

$A_{i}$ : Cross-section area of each beam $\left(\mathrm{m}^{2}\right)$

$\rho_{l_{i}}$ : Linear density of each beam, $7850 A_{i}\left(\mathrm{~kg} \cdot \mathrm{m}^{-1}\right)$

$\tau_{k}:$ Time delay in control (s).

\section{Competing Interests}

The authors declare that they have no competing interests.

\section{Acknowledgments}

The authors would like to gratefully acknowledge the support from Shanghai Sailing Program (16YF1408000), Natural Science Foundation of Shanghai (16ZR1423600), and the National Nature Science Foundation of China (11572224).

\section{References}

[1] Y. Du, R. A. Burdisso, and E. Nikolaidis, "Control of internal resonances in vibration isolators using passive and hybrid dynamic vibration absorbers," Journal of Sound and Vibration, vol. 286, no. 4-5, pp. 697-727, 2005.

[2] H. L. Sun, P. Q. Zhang, H. B. Chen, K. Zhang, and X. L. Gong, "Application of dynamic vibration absorbers in structural vibration control under multi-frequency harmonic excitations," Applied Acoustics, vol. 69, no. 12, pp. 1361-1367, 2008.

[3] T. Mizuno, M. Moriya, and K. Araki, "Robust disturbance cancellation in an active dynamic vibration absorber system," Control Engineering Practice, vol. 3, no. 6, pp. 773-781, 1995.

[4] S. Chatterjee, "Optimal active absorber with internal state feedback for controlling resonant and transient vibration," Journal of Sound and Vibration, vol. 329, no. 26, pp. 5397-5414, 2010.

[5] S.-M. Kim, S. Y. Wang, and M. J. Brennan, "Optimal and robust modal control of a flexible structure using an active dynamic vibration absorber," Smart Materials and Structures, vol. 20, no. 4, Article ID 045003, 2011.

[6] N. D. Anh, H. Matsuhisa, L. D. Viet, and M. Yasuda, "Vibration control of an inverted pendulum type structure by passive mass-spring-pendulum dynamic vibration absorber," Journal of Sound and Vibration, vol. 307, no. 1-2, pp. 187-201, 2007. 
[7] M. Sayed and M. Kamel, "1:2 and 1:3 internal resonance active absorber for non-linear vibrating system," Applied Mathematical Modelling, vol. 36, no. 1, pp. 310-332, 2012.

[8] W. O. Wong and Y. L. Cheung, "Optimal design of a damped dynamic vibration absorber for vibration control of structure excited by ground motion," Engineering Structures, vol. 30, no. 1, pp. 282-286, 2008.

[9] D. H. Kim, J. W. Park, G. S. Lee, and K. I. Lee, "Active impact control system design with a hydraulic damper," Journal of Sound and Vibration, vol. 250, no. 3, pp. 485-501, 2002.

[10] S. Natsiavas, "Steady state oscillations and stability of non-linear dynamic vibration absorbers," Journal of Sound and Vibration, vol. 156, no. 2, pp. 227-245, 1992.

[11] S.-J. Huang, K.-S. Huang, and K.-C. Chiou, "Development and application of a novel radial basis function sliding mode controller," Mechatronics, vol. 13, no. 4, pp. 313-329, 2003.

[12] T. Kobori, H. Kanayama, and S. Kamagata, "Active seismic response control systems for nuclear power plant equipment facilities," Nuclear Engineering and Design, vol. 111, no. 3, pp. 351356, 1989.

[13] J. Xu and K. W. Chung, "Effects of time delayed position feedback on a van der Pol-Duffing oscillator," Physica D, vol. 180, no. 1-2, pp. 17-39, 2003.

[14] Y. Sun and J. Xu, "Experiments and analysis for a controlled mechanical absorber considering delay effect," Journal of Sound and Vibration, vol. 339, pp. 25-37, 2015.

[15] N. Olgac and B. T. Holm-Hansen, "A novel active vibration absorption technique: delayed resonator," Journal of Sound and Vibration, vol. 176, no. 1, pp. 93-104, 1994.

[16] N. Olgae and B. Holm-Hansen, "Design considerations for delayed-resonator vibration absorbers," Journal of Engineering Mechanics, vol. 121, no. 1, pp. 80-89, 1995.

[17] N. Olgac and M. Hosek, "Active vibration absorption using delayed resonator with relative position measurement," Journal of Vibration and Acoustics, vol. 119, no. 1, pp. 131-136, 1997.

[18] Y.-Y. Zhao and J. Xu, "Effects of delayed feedback control on nonlinear vibration absorber system," Journal of Sound and Vibration, vol. 308, no. 1-2, pp. 212-230, 2007.

[19] L. Chen, C. H. Hansen, F. He, and K. Sammut, "Active nonlinear vibration absorber design for flexible structures," International Journal of Acoustics and Vibrations, vol. 12, no. 2, pp. 51-59, 2007.

[20] L. Chen, F. He, and K. Sammut, "Vibration suppression of a principal parametric resonance," Journal of Vibration and Control, vol. 15, no. 3, pp. 439-463, 2009.

[21] K. A. Alhazza and M. A. Majeed, "Free vibrations control of a cantilever beam using combined time delay feedback," Journal of Vibration and Control, vol. 18, no. 5, pp. 609-621, 2012.

[22] C. T. Chatzigogos, A. Pecker, and J. Salençon, "Macroelement modeling of shallow foundations," Soil Dynamics and Earthquake Engineering, vol. 29, no. 5, pp. 765-781, 2009.

[23] A. Abe, Y. Kobayashi, and G. Yamada, "Nonlinear dynamic behaviors of clamped laminated shallow shells with one-to-one internal resonance," Journal of Sound and Vibration, vol. 304, no. 3-5, pp. 957-968, 2007.

[24] J. V. Ferreira, Dynamics response analysis of structures with nonlinear components [Ph.D. thesis], Department of Mechanical Engineering, Imperial College, London, UK, 1998.

[25] A. Raghothama and S. Narayanan, "Bifurcation and chaos in geared rotor bearing system by incremental harmonic balance method," Journal of Sound and Vibration, vol. 226, no. 3, pp. 469-492, 1999.
[26] L. Xu, M. W. Lu, and Q. J. Cao, "Nonlinear vibrations of dynamical systems with a general form of piecewise-linear viscous damping by incremental harmonic balance method," Physics Letters. A, vol. 301, no. 1-2, pp. 65-73, 2002.

[27] J. Xu, K.-W. Chung, and C.-L. Chan, "An efficient method for studying weak resonant double Hopf bifurcation in nonlinear systems with delayed feedbacks," SIAM Journal on Applied Dynamical Systems, vol. 6, no. 1, pp. 29-60, 2007.

[28] J. Xu and K. W. Chung, "A perturbation-incremental scheme for studying Hopf bifurcation in delayed differential systems," Science in China, Series E: Technological Sciences, vol. 52, no. 3, pp. 698-708, 2009.

[29] S. L. Das and A. Chatterjee, "Multiple scales without center manifold reductions for delay differential equations near Hopf bifurcations," Nonlinear Dynamics, vol. 30, no. 4, pp. 323-335, 2002. 


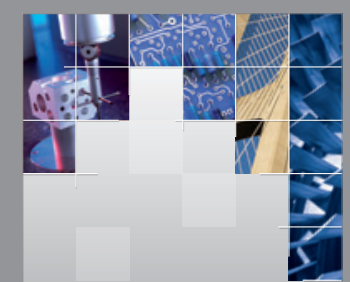

\section{Enfincering}
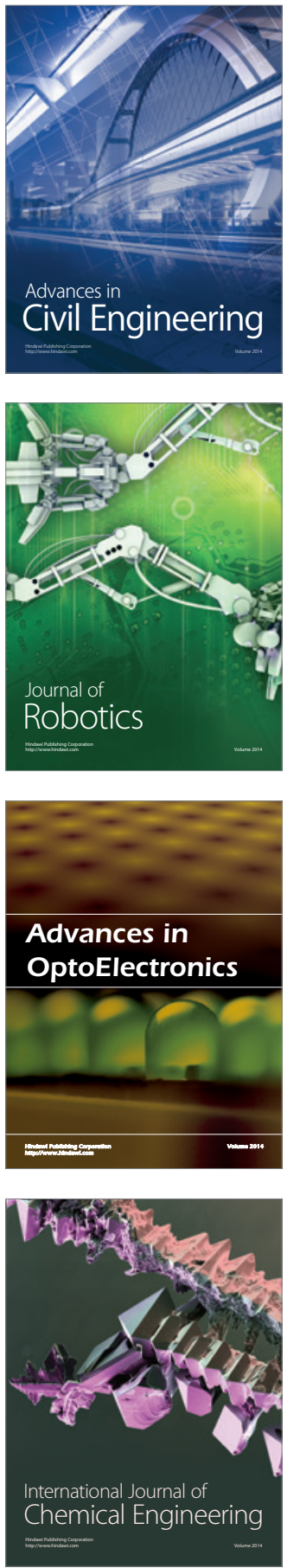

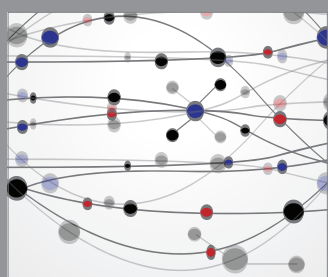

The Scientific World Journal

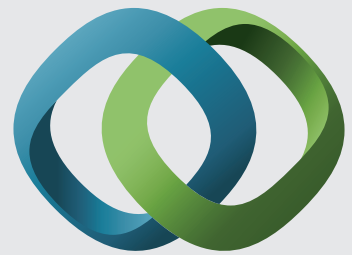

\section{Hindawi}

Submit your manuscripts at

http://www.hindawi.com
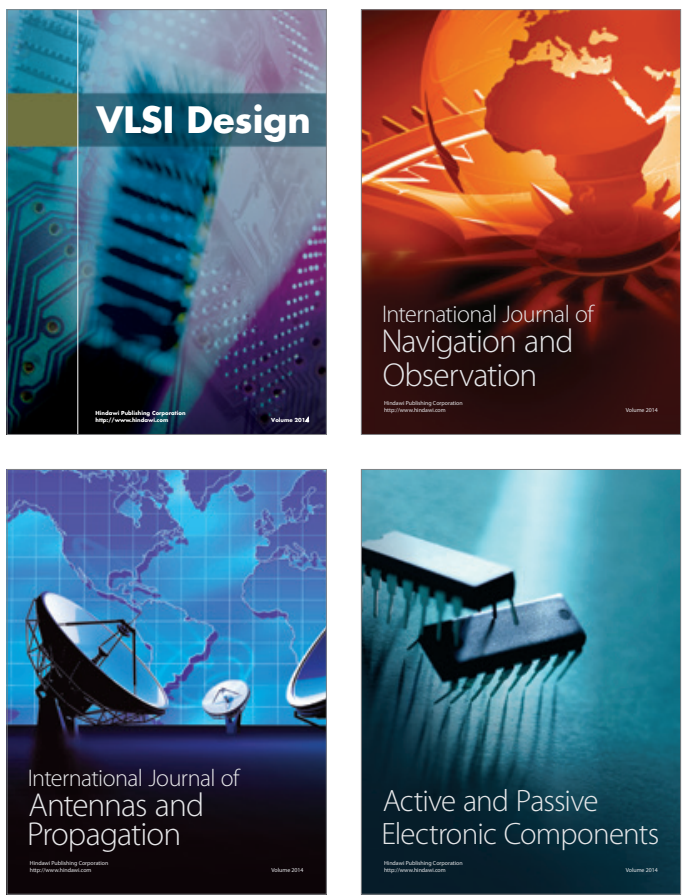
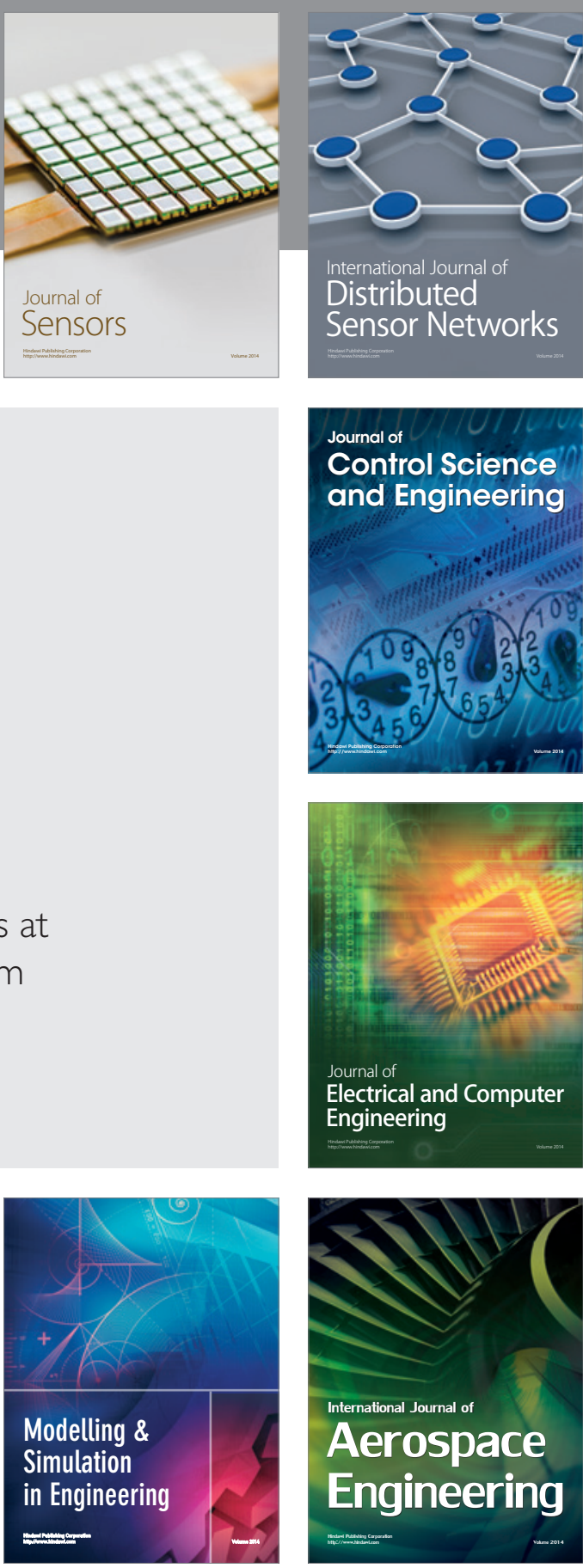

International Journal of

Distributed

Sensor Networks

Journal of

Control Science

and Engineering
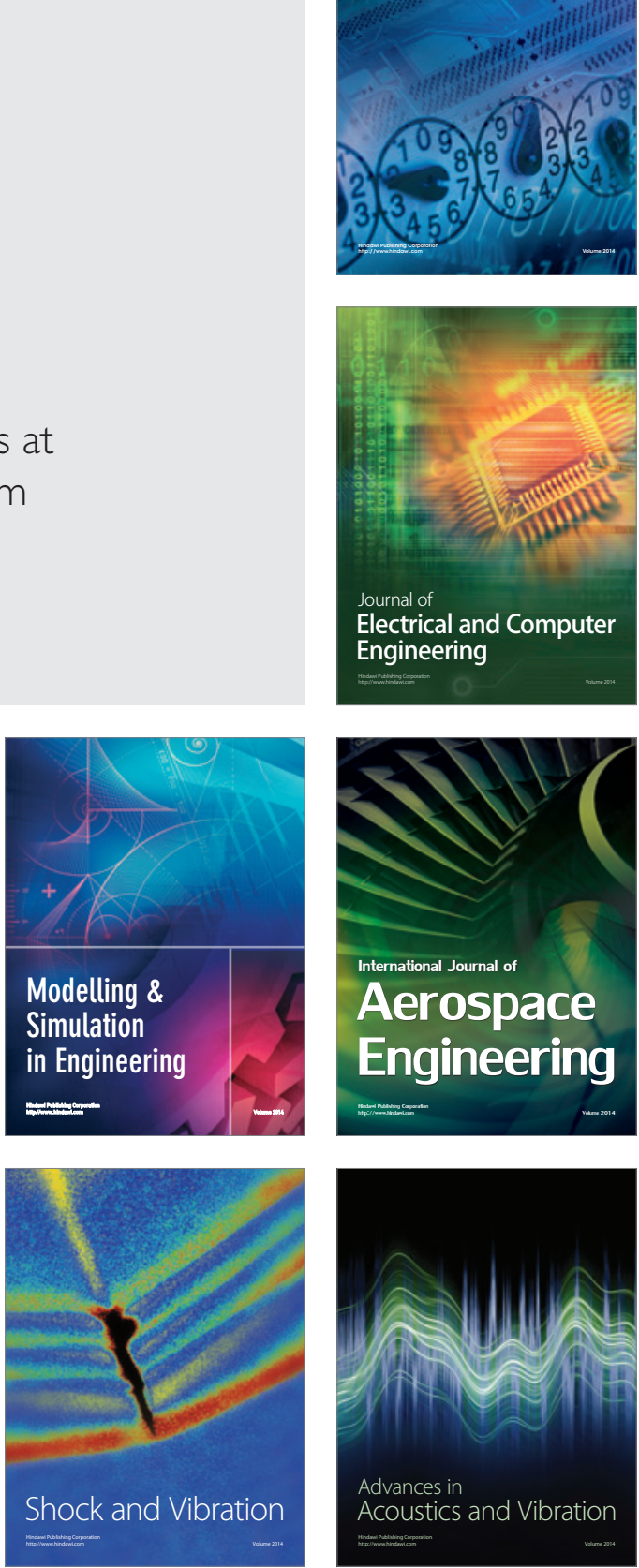